Florida International University FIU Digital Commons

FIU Electronic Theses and Dissertations

University Graduate School

7-15-2002

\title{
D1S80 DNA profiling in five African populations
}

Leslie R. Adrien

Florida International University

DOI: $10.25148 /$ etd.FI13101533

Follow this and additional works at: https://digitalcommons.fiu.edu/etd

Part of the Medical Pathology Commons

\section{Recommended Citation}

Adrien, Leslie R., "D1S80 DNA profiling in five African populations" (2002). FIU Electronic Theses and Dissertations. 1138. https://digitalcommons.fiu.edu/etd/1138

This work is brought to you for free and open access by the University Graduate School at FIU Digital Commons. It has been accepted for inclusion in FIU Electronic Theses and Dissertations by an authorized administrator of FIU Digital Commons. For more information, please contact dcc@fiu.edu. 


\section{FLORIDA INTERNATIONAL UNIVERSITY}

Miami, Florida

D1S80 DNA PROFILING IN FIVE AFRICAN POPULATIONS

A thesis submitted in partial fulfillment of the

requirements for the degree of

MASTER OF SCIENCE

in

FORENSIC SCIENCE

by

Leslie R. Adrien

2002 
To: Dean Arthur W. Herriott

College of Arts and Sciences

This thesis, written by Leslie R. Adrien, and entitled D1S80 DNA Profiling in Five African Populations, having been approved in respect to style and intellectual content, is referred to you for judgment.

We have read this thesis and recommend that it be approved:

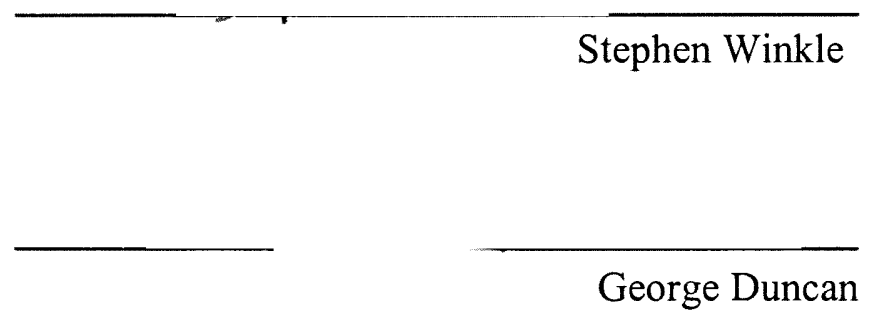

George Duncan

Rene J. Herrera, Major Professor

Date of Defense: July 15, 2002

The thesis of Leslie R. Adrien is approved.

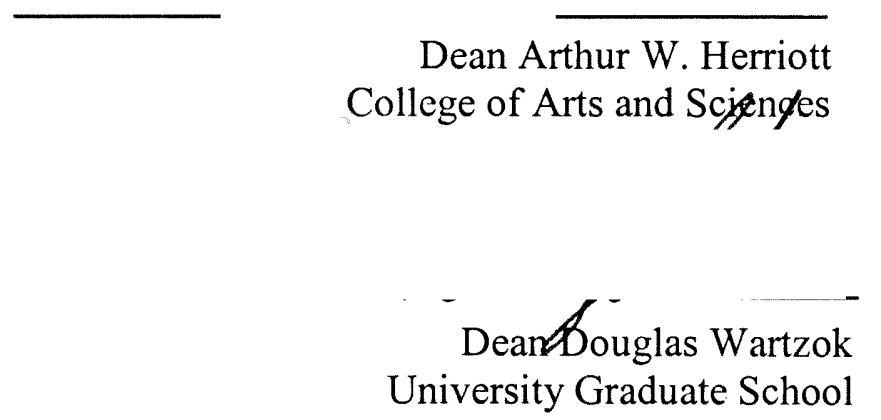

Florida International University, 2002 


\section{DEDICATION}

I dedicate this thesis to my family and friends, whose support and love made it possible to keep my sanity while successfully completing my goals. 


\section{ACKNOWLEDGMENTS}

My deepest indebtedness is to God whose endless grace and blessings enabled me to achieve my current feat.

I would like to thank all the people who have assisted me in accomplishing my objectives. First, to all my lab mates who were always willing to answer my questions and share their technical knowledge with me. I would also like to thank all my committee members for their support.

This research was partially supported by MBRS SCORE GM 08205, MBRS SCORE GM 61347, MARC U-STAR GM 08771. 


\section{ABSTRACT OF THE THESIS \\ D1S80 DNA PROFILING IN FIVE AFRICAN POPULATIONS \\ by}

Leslie R. Adrien

Florida International University, 2002

Miami, Florida

Professor Rene J. Herrera, Major Professor

The highly polymorphic D1S80 locus has no known genetic function. This variable number of tandem repeat (VNTR) has been valuable in forensic identification. We have obtained allelic and genotypic frequencies for five African populations (Benin, Cameroon, Egypt, Kenya and Rwanda), which could be employed as databases to identify individuals.

The polymerase chain reaction, followed by vertical polyacrylamide gel electrophoresis and silver staining was our method of analysis. Allele frequencies were used to infer genetic associations using Phylip 3.5, Principal Component and G-test statistical programs. Tests for Hardy-Weinberg equilibrium were employed. $F_{\text {st }}$ estimates and power of discrimination values were also determined for each of our populations.

Our analyses of 28 additional populations demonstrated that the D1S80 locus alone provided for the discrimination of major racial, groups. Genetic homogeneity between the African groups was observed. We have generated a database useful for human differentiation and phylogenetic studies. 


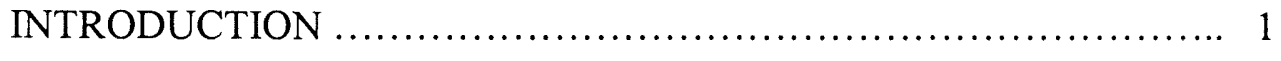

BACKGROUND INFORMATION ................................. 4

MATERIALS AND METHODS

DNA Isolation ............................................... 6

Amplification of DNA Template ........................... 6

Vertical Polyacrylamide Gel Electrophoresis ................... 7

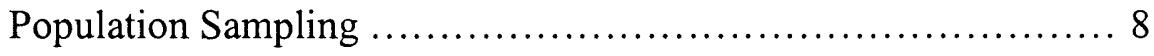

Statistical Analysis ........................................ 11

RESULTS .......................................................... 13

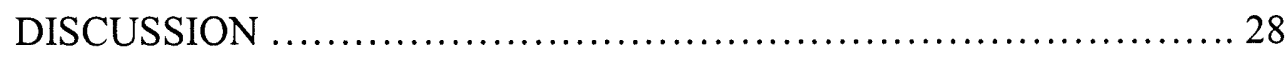

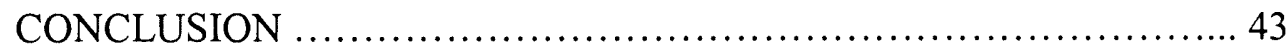

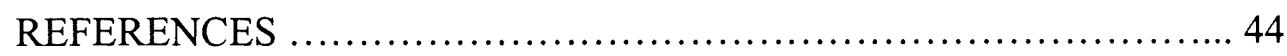




\section{LIST OF TABLES}

TABLE

PAGE

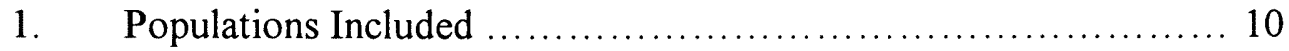

2. Allelic Frequencies ........................................ 14

3. Genotypic Frequencies .................................. 15

4. Observed/Expected Homozygosity and Heterozygosity

Values

5. Test for Hardy-Weinberg Equilibrium and Power of

Discrimination

6. G Scores with probability values between all

33 populations 25 


\section{LIST OF FIGURES}

FIGURE

PAGE

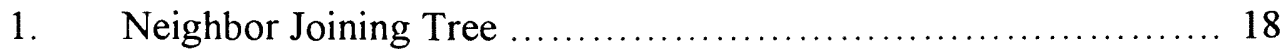

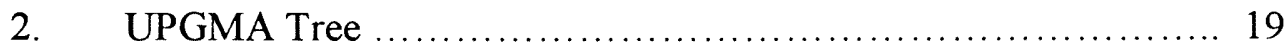

3. Maximum Likelihood Tree with Bootstrap Values .............. 22

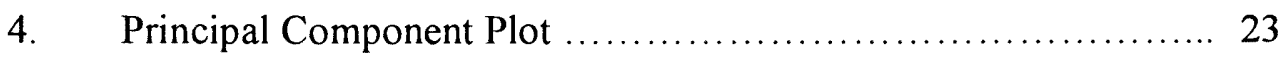

5. Allelic Distribution Graph of the Benin Population .............. 30

6. Allelic Distribution Graph of the Kenya Population .............. 31

7. Allelic Distribution Graph of the Rwanda Population ............. 32

8. Allelic Distribution Graph of the Tanta Population ................ 33

9. Allelic Distribution Graph of the Cameroon2 Population ........... 34

10. Allelic Distribution Graph of the Cameroon Population ........... 35

11. Allelic Distribution Graph of the Congo Population ............... 36

12. Allelic Distribution Graph of the Nigeria Population ............. 37

13. Allelic Distribution Graph of the Zimbabwe Population ........... 38

14. Allelic Distribution Graph of the African American Population .............................................. 39

15. Allelic Distribution Graph of the Caucasian Population ........... 40 


\section{INTRODUCTION}

The D1S80 locus has been utilized in paternity testing, forensic applications and phylogenetic analysis, since its description by Nakamura [1]. It is the most characterized amplified fragment length polymorphism (AmpliFLP) [2]. As a single locus, this variable number of tandem repeat (VNTR) is highly polymorphic. Observed heterozygosity has been reported as high as 78\% [3]. The D1S80 locus by itself enables the discrimination of races and ethnic groups [4]. The standard procedure for D1S80 analysis involves the polymerase chain reaction (PCR) followed by polyacrylamide gel electrophoresis (PAGE) and silver staining. D1S80 is amenable to the PCR since its size ranges from 369 to over 785 base pairs [5].

We decided to focus our study on African populations and their phylogenetic relationships to non-African populations. Sub-Saharan African populations are known for their high levels of diversity [6]. Our research is concentrated on five different populations. These five groups were collected in Benin, Cameroon, Egypt, Kenya and Rwanda. All populations, except the Egyptian population, are sub-Saharan. The majority of all sub-Saharan Africans are now Bantu speaking resulting from the Bantu expansion estimated to have occurred approximately three thousand years ago [6]. The Bantu diaspora was driven by the incipient agricultural revolution in Africa. These farmers/invaders migrated from the central-western coast of Africa eastward and southward. Bantus make up one of four main extant African groups. A brief look into the history of each population provided information on the genetic composition of each. The Sahara desert has been a migration barrier in recent human evolution. Berbers and 
Arabs mainly influenced the genetic make-up of groups in northern Africa, while the heritage south of the Sahara are native Africans, with limited European admixture occurring relatively recently, in the late $15^{\text {th }}$ century [7].

Forty-two separate ethnic groups populate Benin [8]. Due to its location on the west coast of Africa, it was highly involved in the Atlantic slave trade. The natives of this country endured both British and French authority over them for more than a century. The Bantu dispersal is thought to have originated in an area including the country of Benin [6].

Tutsi kings reigned in Rwanda from the late $18^{\text {th }}$ century up to the 1960 's [8]. The Hutus, a Bantu group, penetrated the region west of Lake Victoria during the Bantu dispersal. Ethnic strife exists between these two groups and is manifested through their battles for leadership. Belgium colonized the territory following World War I for a period of approximately 40 years. During this time, the requirement for each tribe to be distinguished with identity cards kept them isolated. Rwanda is known to be one of the most densely populated countries in Africa [9]. The Hutu group makes up $90 \%$ of this country $[8]$.

The native people of Cameroon experienced authority from the Portuguese, British, Germans and French. Cameroon has the most ethnic groups of any other country in Africa, namely 200 [8]. The country is currently divided into a northern French zone and a southern British zone. Cameroon is, geographically, just south of the focus of the Bantu expansion.

Kenya, on the east coast of Africa, has influence of several Arab groups. The cultural and genetic impact of Omani traders is clearly visible in this country. The British 
arrived in the 1800's and assigned different ethnic groups to different parts of the country to live, thereby further isolating these groups. There are more than 100 different ethnic groups established in Kenya [8]. A 1989 census indicated that Arabs, Asians and Europeans made up less than $1 \%$ of the total population [10].

The Egyptian samples were collected from the area of Tanta, which is located on the Nile River delta. This north African population is largely made up of Arabs and Berbers [6]. Berbers were the earliest known inhabitants in north Africa [7]. From 711 of the common era (CE) on, Arabs have dominated the Berbers within these areas [6].

In this study, we examined the D1S80 allelic frequencies of African populations from Benin, Cameroon, Egypt, Kenya and Rwanda and compared them to 28 worldwide reference populations. This information will be the basis of databases for forensic probability estimation and population genetic studies. Allelic and genotypic frequencies were determined by utilizing the gene count method [11]. Divergence from HardyWeinberg equilibrium (HWE) expectations were determined, with the Fisher exact test and the Chi-Square test, as well as $\mathrm{F}_{s t}$ estimates and power of discrimination values in each of our populations. Observed and expected homozygosity and heterozygosity values were also generated. In addition, twenty-eight other populations were incorporated into our study to ascertain possible genetic similarities. Allele frequencies were used to infer genetic associations through phylogenetic, principal component and GTest statistical analyses. 


\section{BACKGROUND INFORMATION}

The D1S80 locus is located at the telomeric position of the $\mathrm{p}$ arm of chromosome

1. Nakamura and collaborators first described this locus in 1988 [3]. Its designation stems from the fact that this is a $\underline{D} N A$ locus on chromosome $\underline{1}$, a $\underline{\text { Single copy sequence }}$ and it was the $\underline{80}^{\text {th }}$ locus described on chromosome 1. D1S80 is a VNTR that is also referred to as an AmplifLP. To date, no phenotypic trait has been associated with this locus [5]. AmplifLPs are distinguished by differences in length between the alleles, not by sequence $[3,12]$, although considerable sequence differences have been reported. The overall length ranges of AmpliFLPs are from 200 base pairs (bp) to $1000 \mathrm{bp}$ [3]. The D1S80 known allele size range, including the flanking regions, is from $369 \mathrm{bp}$ to $785 \mathrm{bp}$ [5]. D1S80 has a core repeat unit of 16 bps [3]. D1S80 alleles typically contain from 14 to 41 repeats of this 16 bp sequence [13]. D1S80 alleles are co-dominantly inherited [12]. Estimates of heterozygosity are generally greater than $70 \%[14,15]$. Although this is a single locus, it is highly polymorphic due to the large number of alleles it contains (more than 27). As many as twenty-nine different alleles have been found within one population $[5,13]$. The number of possible genotypes can be calculated by the formula $\mathrm{G}$ $=\mathrm{n}(\mathrm{n}+1) / 2[3]$. According to this formula, 435 genotypes are possible when 29 alleles are identified. A high number of alleles most likely result from high mutation rates and a lack of selection pressure. Alleles with greater than 41 repeats and those smaller than 14 have both been reported [16-19]. The alleles with the highest recurring frequency are those with 18 and 24 repeats $[12,20-22]$. The high frequency of these alleles suggests 
that these are ancestral alleles [23]. Allele 15 is not common, and therefore is not incorporated into D1S80 allelic ladders. 


\section{MATERIALS AND METHODS}

\section{DNA Isolation}

All samples were collected as whole blood in EDTA Vacutainer tubes from unrelated individuals. These individuals were identified by biographical information traced back at least two generations. The appropriate ethical guidelines, as dictated by Florida International University, were followed during the collection of samples. Each collection was arranged through the leaders of the regions and supervised by the same. The blood samples were lysed and leukocyte nuclei were separated from the rest of the blood components. Several washes in lysis buffer were performed to rinse and isolate the leukocyte nuclei. The DNA was then purified using proteinase-K digestion and standard organic phenol-chloroform extraction. Ethanol precipitation followed to pellet the DNA. DNA was then dissolved in TE (Tris-Ethylenediamine Tetraacetate) buffer. All samples were stored in $-80^{\circ} \mathrm{C}$ when not in use.

\section{Amplification of DNA}

PCR was performed in a Perkin-Elmer 480 thermal cycler. Amplification parameters were 1 minute at $94^{\circ} \mathrm{C}, 1$ minute at $65^{\circ} \mathrm{C}$ and 1 minute at $72^{\circ} \mathrm{C}$ for twenty-nine cycles. An additional 10-minute extension at $72^{\circ} \mathrm{C}$ followed [13]. PCR products were stored at $4^{\circ} \mathrm{C}$ until they were loaded onto a polyacrylamide gel. PCR amplifications were carried out in $25 \mu 1$ reactions containing $16.3 \mu 1$ of water, $2.5 \mu 1$ of $10 \mathrm{X}$ buffer containing 
$15 \mathrm{mM} \mathrm{MgCl}_{2}, 2.5 \mu \mathrm{l}(0.15 \mathrm{mM}) \mathrm{dNTPs}, 1.25 \mu \mathrm{l}(10 \mu \mathrm{M})$ of each primer, $0.2 \mu \mathrm{l}(5 \mathrm{U}) \mathrm{Taq}$ DNA and 10-50 nanograms of DNA sample. The sequence of the forward primer was 5', GAAACTGGCCTCCAAACACTGCCCGCCG 3' and that of the reverse primer was 5' GTCTTGTTGGAGATGCACGTGCCCCTTGC 3' [24]. The Benin samples were DOP (degenerate oligonucleotide primer) pre-amplified according to Buchanan and collaborators [25]. This method of PCR is used when the DNA is of poor quality or low yield. From the DOP-PCR product, $6 \mu$ l were used to specifically amplify for D1S80. Samples were overlaid with two drops of mineral oil to protect them from evaporation throughout the amplification procedure.

\section{Vertical Polyacrylamide Gel Electrophoresis}

A 39:1 acrylamide stock solution was used to make an $8 \%$ polyacrylamide gel. The longer glass plate used for casting the slab gel was treated with an adherent to retain the gel as the shorter plate is detached. Gels were $35 \times 45$ centimeters in size with a 0.8 millimeter thickness or $38 \times 46$ centimeters in size with a 1-millimeter thickness. An Applied Biosystems AmpliFLP D1S80 Allelic Ladder was electrophoresed every four samples. The gels were run at 1000-1200 volts for five to six hours, depending upon the migration of the xylene-cyanol tracking dye. Electrophoresis was stopped 45 minutes after the xylene-cyanol dye ran out of the bottom of the gel. All gels were silver stained to visualize the alleles.

Silver staining consisted of a 5-minute slow agitation in $1 \%$ nitric acid. Following a water rinse, the gel was placed in fresh $0.2 \%$ silver nitrate and shaken for 20 
minutes. Another water rinse was done prior to the development step in fresh $0.28 \mathrm{M}$ sodium carbonate, $0.05 \%$ formaldehyde solution. Development consisted of several rinses in the sodium carbonate solution until it no longer became "milky". Once the desired band intensities were achieved, the gel was placed in $10 \%$ acetic acid stop solution for 5 minutes. Gels were then placed in water for another 5 minutes. After drying, gels were photographed under visible light. All gels were scored to ascertain genotypes independently by at least two people. Genotyping was performed directly from the gels by comparison to the allelic ladder. Any discrepancies were repeated.

\section{Population Sampling}

A total of five African populations were analyzed in our study. They were Benin, Cameroon, Egypt, Kenya and Rwanda. All the sub-Saharan populations belong to the Niger-Congo language family. A total of one hundred samples were analyzed from Benin. These individuals belong to the Fon ethnic group. They were collected in the southern part of the country from the Zagnanado population. The Kenya group consisted of 106 Bantu individuals from small villages 100 kilometers northeast of Nairobi. The Rwanda population was made up of 100 individuals of the Hutu tribe. Hutus are a Bantu group. The Egyptian group was collected in the region of Tanta located at the center of the Nile delta. Tanta is approximately 94 kilometers north of Egypt's capital city, Cairo. This population is primarily Arab/Berber in origin. Arabia and north Africa both share the Afro-Asiatic language. This north African population shares their ancestry to a lesser extent with Greeks, Turks and other Mediterranean Basin people. Tantas are therefore 
Caucasians. There were 100 samples from Tanta processed. The Cameroon group consisted of 16 Bantu individuals from villages 50 kilometers southwest of the city of Yaounde, in the southern region of the country. In our study, these 16 samples were analyzed together with the 34 individuals reported by Araujo da Silva in 1999 [26]. Another twenty-eight populations were analyzed along with these in order to ascertain genetic relationships. These are all extracted from published information as indicated in Table 1. 
Table 1. Populations included

\begin{tabular}{|c|c|c|c|c|}
\hline Population & $\begin{array}{c}3 \text { Letter } \\
\text { Code* }\end{array}$ & $\mathbf{N}$ & Location & Reference \\
\hline Andalucia & Anc & 120 & Southern province of Spain & {$[27]$} \\
\hline Andalusian & Ans & 147 & Southern province of Spain & {$[16]$} \\
\hline ArabMoslem & Ara & 94 & Gaza Strip, Judaea, Samaria, Israel & {$[28]$} \\
\hline Australia & Aus & 250 & Victoria & {$[29]$} \\
\hline Bahama & Bah & 88 & Throughout Bahamas & {$[4]$} \\
\hline Bari & Bar & 24 & NE Colombia, S. America & {$[4]$} \\
\hline Basque & Bas & 257 & North Central Spain & {$[28]$} \\
\hline Benin & Ben & 100 & S. Benin, West Africa & Present Study \\
\hline BWHAlaska & BWH & 109 & Bethel-Wade Hampton, Alaska & {$[30]$} \\
\hline Cameroon & Cam & 34 & Yaounde City & {$[26]$} \\
\hline Cameroon2 & $\mathrm{Ca} 2$ & 16 & SW of Yaounde City, W. Africa & Present Study \\
\hline CanaryIsl & Can & 123 & General population from Canary Islands & {$[16]$} \\
\hline Chimila & $\mathrm{Chm}$ & 46 & NE Colombia, S. America & {$[4]$} \\
\hline China (Han) & Chn & 216 & Xian \& Shijiazhuang districts of China & {$[28]$} \\
\hline Congo & Con & 34 & Lubumbashi City & {$[26]$} \\
\hline Denmark & Den & 210 & General population from Denmark & {$[28]$} \\
\hline DubaiArab & Dub & 93 & United Arab Emirates & {$[31]$} \\
\hline Egypt & Tan & 100 & Tanta, N. Africa & Present Study \\
\hline Galicia & Gal & 149 & NW province of Spain & {$[28]$} \\
\hline Greece & Gre & 107 & Cyprus & {$[32]$} \\
\hline Haiti & Hai & 83 & Caribbean & [28] \\
\hline Kenya & Ken & 106 & NE of Nairobi, E. Africa & Present Study \\
\hline Korea & Kor & 116 & Seoul & {$[28]$} \\
\hline MapucheArg & Map & 61 & Argentina, S. America & [33] \\
\hline Navajo & Nav & 28 & New Mexico, USA & {$[4]$} \\
\hline Nigeria & $\mathrm{Nig}$ & 67 & W. Africa & {$[28]$} \\
\hline N.SlopeAlas & NSA & 92 & North Slope Borough, Alaska & {$[30]$} \\
\hline Philippines & Phi & 103 & Metro Manila & [34] \\
\hline Rwanda & Rwa & 100 & E. Africa & Present Study \\
\hline SaudiArabia & $\mathrm{Sau}$ & 220 & Riyadh & {$[35]$} \\
\hline Taiwan (Han) & Tai & 105 & General population of Taiwan & {$[28]$} \\
\hline Turkey & Tur & 112 & General population of Turkey & {$[36]$} \\
\hline Zimbabwe & Zim & 101 & Mashonaland Province & {$[28]$} \\
\hline
\end{tabular}

*These codes are used in Table 6. 


\section{Statistical Analysis}

The gene counting method [11] was used to generate genotypic and allelic frequencies. Allelic frequencies were analyzed with PHYLIP 3.5 [37], Numerical Taxonomy and Multivariate Analysis System (NTSys) principal component [38], and Carmody's G-test [39]. The depiction of homogeneic clustering within PHYLIP and NTSys suggests adequate population sampling. The SEQBOOT, CONSENSE, GENDIST, CONTML, UPGMA, NJ and ML programs are all within the PHYLIP software. Phylogenetic trees were used to visualize the general phylogenetic relationships between the populations and as a proof of the integrity of the data. Three distance-based analyses were used to generate dendrograms, the Unweighted Pair Group Method with Averages (UPGMA), Neighbor Joining (NJ) and Maximum Likelihood (ML). UPGMA assumes that the rates of mutation are equal across time for all lineages [40]. Nei genetic distances were calculated through the use of the GENDIST program. This tree was then created with the UPGMA program, 1000 bootstrap replications were generated with SEQBOOT and CONSENSE configured a consensus tree. The NJ method, on the other hand, produces an un-rooted network. When rooted, a constant rate of evolution is assumed. First, GENDIST was used to calculate Nei's genetic distances. The NJ program was then run, SEQBOOT generated 1000 bootstrap replications and a consensus tree was built with CONSENSE. The NJ method offers rapid analysis with large data sets. The final tree was done with the ML method, which does not assume constant evolutionary rates, thereby enhancing the validity of the relationships in the other trees. SEQBOOT produced a large number (1000) of bootstrapped data sets that 
were then inputted into the CONTML program. The CONTML and CONSENSE programs configured the best-fit tree directly from the allele frequencies. The principal component (PC) test was performed to generate a two dimensional plot of PC 1 and 2 . In PC analysis, allelic frequencies are inputted into the NTSys program to segregate and cluster groups within by plotting according to their variability. PCs 1 and 2 usually contain the most amount of variability, with subsequent PCs decreasing in value. The Gtest calculates likelihood ratios according to the obtained frequencies. It contrasts the observed and expected frequencies [15]. One thousand simulations were done for each 2 x 2 contingency table. The G-test analyzes each $2 \times 2$ table and indicates which populations are homogeneous with each other. Probability values less than 0.050 indicate significantly different populations.

In addition to these phylogenetic and statistical analyses, several other parameters were computed to describe the five African populations. Expected homozygosity and heterozygosity values were compared to those reported in the literature. Conformance to Hardy-Weinberg equilibrium was tested by use of the Fisher exact and Chi-Square tests within the Genetic Data Analysis (GDA) software [41]. This software was also used to calculate theta-P values which are equivalent to $F_{\mathrm{st}}$ values [41]. This analysis estimates the probability that two alleles are identical by descent [42]. It is an attempt to offset the substructure effects on heterozygote genotypic frequencies [43]. Assumptions are no mutation rate and the sampling of an effective population size. If these assumptions are met, these values range between 0 and 1 . This measure indirectly indicates gene flow [44]. 


\section{RESULTS}

Table 2 shows the allelic frequencies for the five African populations. The 24 allele was most frequent in the Benin, Kenya, Rwanda and Tanta populations with frequencies of $0.2150,0.2028,0.2200$ and 0.4150 , respectively. In the Cameroon population the 34 allele was most common with a frequency of 0.3125 . African groups have been shown to have the highest frequencies for the 24, 28 and 34 alleles [45]. All five populations shared alleles $17,20,21,23,24,25,28,31$ and 34 . Only within the Rwandan population was allele 16 detected and allele 36 was found in a single Cameroon sample.

There were 16 different alleles and 50 different genotypes found in the Benin population. Within the Kenyan and Rwandan groups, 17 various alleles and 49 various genotypes were distinguished. The Tanta samples exhibited 18 alleles and 40 genotypes. There were 11 alleles and 14 genotypes discerned in the Cameroon samples. All genotypic frequencies are contained in Table 3 . Within the Benin population, the 22/24 genotype was most frequent representing $6 \%$ of the samples. In the Kenya population, $24 / 28$ was the most recurring genotype representing $7.55 \%$ of the total. Both $24 / 34$ and $17 / 24$ were most abundant within the Rwandan samples, each with a frequency of $6 \%$. The homozygous genotype, 24/24, was highly repetitive within the Tanta population, displaying a frequency of $18 \%$. Cameroon also had two most recurrent genotypes. These were $21 / 34$ and $25 / 34$, both with a frequency of $12.5 \%$. The number of alleles and genotypes observed were not exceptionally high compared to the number of genotypes that are possible. Utilizing the formula $G=n(n+1) / 2$, the most genotypes attainable for 


\begin{tabular}{|c|c|c|c|c|c|}
\hline Alleles & Benin $(N=100)$ & $\begin{array}{c}\text { Kenya } \\
(\mathrm{N}=106)\end{array}$ & $\begin{array}{l}\text { Rwanda } \\
(\mathrm{N}=100)\end{array}$ & $\begin{array}{c}\text { Tanta } \\
(\mathrm{N}=100)\end{array}$ & $\begin{array}{c}\text { Cameroon } 2 \\
(\mathrm{~N}=16)\end{array}$ \\
\hline 14 & $\cdots$ & $\ldots$ & & $\cdots$ & $\ldots$ \\
\hline 15 & $\ldots$ & $\ldots$ & $\ldots$ & $\ldots$ & $\ldots$ \\
\hline 16 & $\ldots$ & $\ldots$ & 0.0100 & $\ldots$ & .. \\
\hline 17 & 0.0550 & 0.0283 & 0.1050 & 0.0150 & 0.0625 \\
\hline 18 & 0.0550 & 0.0613 & 0.0900 & 0.1400 & $\cdots$ \\
\hline 19 & & $\ldots$ & 0.0050 & 0.0050 & $\ldots$ \\
\hline 20 & 0.0550 & 0.0236 & 0.0100 & 0.0150 & 0.0313 \\
\hline 21 & 0.1100 & 0.0991 & 0.1000 & 0.0300 & 0.1563 \\
\hline 22 & 0.1000 & 0.0896 & 0.1200 & 0.0450 & $\ldots$ \\
\hline 23 & 0.0300 & 0.0283 & 0.0050 & 0.0350 & 0.0313 \\
\hline 24 & 0.2150 & 0.2028 & 0.2200 & 0.4150 & 0.2188 \\
\hline 25 & 0.0650 & 0.0377 & 0.0300 & 0.0600 & 0.0625 \\
\hline 26 & 0.0050 & 0.0142 & $\ldots$ & 0.0300 & $\cdots$ \\
\hline 27 & 0.0100 & 0.0236 & 0.0550 & 0.0200 & $\ldots$ \\
\hline 28 & 0.1100 & 0.1981 & 0.0700 & 0.0650 & 0.0313 \\
\hline 29 & 0.0200 & 0.0330 & 0.0100 & 0.0500 & $\cdots$ \\
\hline 30 & 0.0100 & $\ldots$ & $\ldots$ & 0.0050 & $\ldots$ \\
\hline 31 & 0.0800 & 0.0330 & 0.0400 & 0.0400 & 0.0313 \\
\hline 32 & 0.0050 & 0.0283 & 0.0200 & 0.0050 & $\cdots$ \\
\hline 33 & $\ldots$ & 0.0047 & $\ldots$ & 0.0050 & 0.0313 \\
\hline 34 & 0.0750 & 0.0802 & 0.1000 & 0.0200 & 0.3125 \\
\hline 35 & $\cdots$ & $\ldots$ & $\ldots$ & $\ldots$ & $\cdots$ \\
\hline 36 & $\ldots$ & $\cdots$ & $\ldots$ & $\cdots$ & 0.0313 \\
\hline 37 & $\ldots$ & $\ldots$ & $\ldots$ & $\ldots$ & $\ldots$ \\
\hline 38 & $\ldots$ & $\ldots$ & $\ldots$ & $\ldots$ & $\cdots$ \\
\hline 39 & $\ldots$ & $\ldots$ & $\cdots$ & $\cdots$ & $\cdots$ \\
\hline 40 & $\ldots$ & 0.0142 & 0.0100 & $\ldots$ & $\ldots$ \\
\hline 41 & $\ldots$ & $\ldots$ & $\ldots$ & $\ldots$ & $\ldots$ \\
\hline
\end{tabular}

$\ldots=$ allele not detected; $\mathrm{N}=$ total number of individuals

Theta-P values: $B$ enin $=0.032$, Kenya $=0.027$, Rwanda $=0.029$, Tanta $=0.009$, Cameroon $2=0.021$ 
Table 3. Genotypic Frequencies

\begin{tabular}{|c|c|c|c|c|c|c|c|c|c|c|c|c|c|c|c|c|c|c|c|c|c|}
\hline \multirow[t]{2}{*}{ Genotype } & \multicolumn{2}{|c|}{ Benin } & \multicolumn{2}{|c|}{ Kenya } & \multicolumn{2}{|c|}{ Rwanda } & \multicolumn{2}{|c|}{ Tanta } & \multicolumn{2}{|c|}{ Cameroon2 } & \multirow[t]{2}{*}{ Genotype } & \multicolumn{2}{|c|}{ Benin } & \multicolumn{2}{|c|}{ Kenya } & \multicolumn{2}{|c|}{ Rwanda } & \multicolumn{2}{|c|}{ Tanta } & \multicolumn{2}{|c|}{ Cameroon2 } \\
\hline & $\mathbf{N}$ & Freq & $\mathrm{N}$ & Freq & $\mathrm{N}$ & Freq & $\mathbf{N}$ & Freq & $\mathbf{N}$ & Freq & & $\mathbf{N}$ & Freq & $\mathbf{N}$ & Freq & $\mathbf{N}$ & Freq & $\mathrm{N}$ & Freq & $\mathbf{N}$ & Freq \\
\hline $16 / 16$ & & $\ldots$ & & $\ldots$ & 1 & 0.010 & & $\ldots$ & & $\ldots$ & $20 / 24$ & 2 & 0.0200 & 2 & 0.018 & 1 & 0.0100 & 2 & 0.0200 & & $\ldots$ \\
\hline $17 / 17$ & 3 & 0.0300 & & $\cdots$ & 3 & 0.030 & & $\cdots$ & & $\ldots$ & $20 / 25$ & 2 & 0.0200 & & & & $\ldots$ & & $\ldots$ & & $\ldots$ \\
\hline $17 / 18$ & & $\cdots$ & & $\cdots$ & 1 & 0.010 & & $\cdots$ & & $\cdots$ & $20 / 29$ & 1 & 0.0100 & & & & $\ldots$ & 1 & 0.0100 & & $\ldots$ \\
\hline $17 / 21$ & & $\ldots$ & & $\cdots$ & 3 & 0.030 & & $\cdots$ & & $\ldots$ & $20 / 31$ & 1 & 0.0100 & 1 & 0.009 & & $\ldots$ & & $\ldots$ & & $\ldots$ \\
\hline $17 / 22$ & 2 & 0.0200 & 1 & 0.0094 & 2 & 0.020 & & $\cdots$ & & $\cdots$ & $20 / 32$ & 1 & 0.0100 & & & & $\ldots$ & & $\ldots$ & & $\ldots$ \\
\hline $17 / 24$ & 1 & 0.0100 & 2 & 0.0189 & 6 & 0.060 & 2 & 0.0200 & 1 & 0.0625 & $20 / 33$ & & $\ldots$ & & & & $\ldots$ & & $\ldots$ & 1 & 0.0625 \\
\hline $17 / 25$ & & $\ldots$ & & $\ldots$ & 1 & 0.010 & & $\ldots$ & & $\ldots$ & $20 / 34$ & & $\ldots$ & 2 & 0.018 & & $\ldots$ & & $\ldots$ & & $\ldots$ \\
\hline $17 / 28$ & & $\cdots$ & 2 & 0.0189 & & & & $\ldots$ & & $\ldots$ & $21 / 21$ & 1 & 0.0100 & & & & $\ldots$ & 1 & 0.0100 & 1 & 0.0625 \\
\hline $17 / 29$ & & $\ldots$ & 1 & 0.0094 & & & 1 & 0.0100 & & $\ldots$ & $21 / 22$ & 3 & 0.0300 & 3 & 0.028 & 5 & 0.0500 & & $\ldots$ & & $\ldots$ \\
\hline $17 / 31$ & 1 & 0.0100 & & $\ldots$ & & & & $\ldots$ & & $\ldots$ & $21 / 24$ & 5 & 0.0500 & 5 & 0.047 & 5 & 0.0500 & & $\ldots$ & 1 & 0.0625 \\
\hline $17 / 34$ & 1 & 0.0100 & & $\ldots$ & 2 & 0.020 & & $\ldots$ & 1 & 0.0625 & $21 / 25$ & & $\ldots$ & 2 & 0.018 & & $\ldots$ & & $\ldots$ & & $\ldots$ \\
\hline $18 / 18$ & 1 & 0.0100 & & $\ldots$ & 2 & 0.020 & 5 & 0.0500 & & $\ldots$ & $21 / 27$ & & $\ldots$ & 1 & 0.009 & & $\ldots$ & & $\cdots$ & & $\ldots$ \\
\hline $18 / 21$ & & $\ldots$ & 2 & 0.0189 & 2 & 0.020 & & $\ldots$ & & $\ldots$ & $21 / 28$ & 5 & 0.0500 & 6 & 0.056 & 2 & 0.0200 & & $\ldots$ & & $\ldots$ \\
\hline $18 / 22$ & & $\ldots$ & 1 & 0.0094 & 3 & 0.030 & & $\ldots$ & & $\ldots$ & $21 / 29$ & & $\ldots$ & & & & $\ldots$ & 2 & 0.0200 & & $\ldots$ \\
\hline $18 / 23$ & 1 & 0.0100 & & $\ldots$ & & & & $\ldots$ & & $\ldots$ & $21 / 31$ & 1 & 0.0100 & & & & $\ldots$ & 1 & 0.0100 & & $\ldots$ \\
\hline $18 / 24$ & 4 & 0.0400 & 3 & 0.0283 & 4 & 0.040 & 11 & 0.1100 & & $\ldots$ & $21 / 32$ & & $\ldots$ & & & 1 & 0.0100 & & $\ldots$ & & $\ldots$ \\
\hline $18 / 25$ & 1 & 0.0100 & 1 & 0.0094 & & & 2 & 0.0200 & & $\ldots$ & $21 / 34$ & 5 & 0.0500 & 2 & 0.018 & 2 & 0.0200 & 1 & 0.0100 & 2 & 0.1250 \\
\hline $18 / 27$ & & $\ldots$ & & $\ldots$ & 1 & 0.010 & 1 & 0.0100 & & $\ldots$ & $22 / 22$ & 3 & 0.0300 & & & 1 & 0.0100 & & $\ldots$ & & $\ldots$ \\
\hline $18 / 28$ & 2 & 0.0200 & 4 & 0.0377 & & & 3 & 0.0300 & & $\ldots$ & $22 / 23$ & & $\ldots$ & 1 & 0.009 & & $\ldots$ & & $\ldots$ & & $\ldots$ \\
\hline $18 / 29$ & & $\ldots$ & & $\ldots$ & & & 1 & 0.0100 & & $\ldots$ & $22 / 24$ & 6 & 0.0600 & 2 & 0.018 & 4 & 0.0400 & 4 & 0.0400 & & $\ldots$ \\
\hline $18 / 31$ & 1 & 0.0100 & & $\ldots$ & 1 & 0.010 & & $\ldots$ & & $\ldots$ & $22 / 25$ & 1 & 0.0100 & & & & $\ldots$ & 2 & 0.0200 & & $\cdots$ \\
\hline $18 / 32$ & & $\ldots$ & 1 & 0.0094 & & & & $\ldots$ & & $\ldots$ & $22 / 27$ & & $\ldots$ & & & 3 & 0.0300 & 1 & 0.0100 & & $\ldots$ \\
\hline $18 / 34$ & & $\ldots$ & 1 & 0.0094 & 2 & 0.020 & & $\ldots$ & & $\ldots$ & $22 / 28$ & 1 & 0.0100 & 5 & 0.047 & 1 & 0.0100 & & $\ldots$ & & $\ldots$ \\
\hline $19 / 22$ & & $\ldots$ & & $\ldots$ & 1 & 0.010 & & $\ldots$ & & $\ldots$ & $22 / 29$ & & $\ldots$ & 2 & 0.018 & & $\ldots$ & & $\ldots$ & & $\ldots$ \\
\hline $19 / 24$ & & $\ldots$ & & $\ldots$ & & & 1 & 0.0100 & & $\ldots$ & $22 / 31$ & & $\ldots$ & 2 & 0.018 & & $\ldots$ & 2 & 0.0200 & & $\ldots$ \\
\hline $20 / 20$ & 1 & 0.0100 & & $\ldots$ & & & & $\ldots$ & & $\ldots$ & $22 / 32$ & & $\ldots$ & & & 1 & 0.0100 & & $\ldots$ & & $\ldots$ \\
\hline $20 / 21$ & 1 & 0.0100 & & $\ldots$ & & & & $\ldots$ & & $\ldots$ & $22 / 34$ & & $\ldots$ & 2 & 0.018 & 1 & 0.0100 & & $\ldots$ & & $\ldots$ \\
\hline $20 / 22$ & 1 & 0.0100 & & $\ldots$ & 1 & 0.010 & & $\ldots$ & & $\ldots$ & $23 / 23$ & 1 & 0.0100 & & & & $\ldots$ & & $\ldots$ & & $\ldots$ \\
\hline
\end{tabular}


Table 3. Genotypic Frequencies (Con't)

\begin{tabular}{|c|c|c|c|c|c|c|c|c|c|c|c|c|c|c|c|c|c|c|c|c|c|}
\hline \multirow[t]{2}{*}{ Genotype } & \multicolumn{2}{|c|}{ Benin } & \multicolumn{2}{|c|}{ Kenya } & \multicolumn{2}{|c|}{ Rwanda } & \multicolumn{2}{|c|}{ Tanta } & \multicolumn{2}{|c|}{ Cameroon2 } & \multirow[t]{2}{*}{ Genotype } & \multicolumn{2}{|c|}{ Benin } & \multicolumn{2}{|c|}{ Kenya } & \multicolumn{2}{|c|}{ Rwanda } & \multicolumn{2}{|c|}{ Tanta } & \multicolumn{2}{|c|}{ Cameroon2 } \\
\hline & $\mathbf{N}$ & Freq & $\mathbf{N}$ & Freq & $\mathbf{N}$ & Freq & $\mathrm{N}$ & Freq & $\mathbf{N}$ & Freq & & $\mathbf{N}$ & Freq & $\mathrm{N}$ & Freq & $\mathbf{N}$ & Freq & $\mathbf{N}$ & Freq & $\mathbf{N}$ & Freq \\
\hline $23 / 31$ & & $\ldots$ & 1 & 0.0094 & 1 & 0.0100 & & $\ldots$ & & $\ldots$ & $26 / 31$ & & $\ldots$ & & & & $\ldots$ & 1 & 0.0100 & & $\ldots$ \\
\hline $23 / 32$ & & $\ldots$ & 1 & 0.0094 & & $\ldots$ & & $\ldots$ & & $\ldots$ & $27 / 27$ & & $\ldots$ & & & 1 & 0.0100 & 1 & 0.0100 & & $\ldots$ \\
\hline $23 / 34$ & 1 & 0.0100 & 1 & 0.0094 & & $\ldots$ & & $\ldots$ & & $\ldots$ & $27 / 33$ & & $\ldots$ & 1 & 0.009 & & $\ldots$ & & $\ldots$ & & $\ldots$ \\
\hline $24 / 24$ & 2 & 0.0200 & 3 & 0.0283 & 3 & 0.0300 & 18 & 0.1800 & 1 & 0.0625 & $27 / 34$ & 1 & 0.0100 & 1 & 0.009 & 2 & 0.0200 & & $\ldots$ & & $\ldots$ \\
\hline $24 / 25$ & 2 & 0.0200 & 3 & 0.0283 & 2 & 0.0200 & 4 & 0.0400 & & $\ldots$ & $28 / 28$ & 1 & 0.0100 & 4 & 0.037 & 2 & 0.0200 & 1 & 0.0100 & & $\ldots$ \\
\hline $24 / 26$ & 1 & 0.0100 & 2 & 0.0189 & & $\ldots$ & 2 & 0.0200 & & $\ldots$ & $28 / 29$ & & $\ldots$ & 3 & 0.028 & & $\ldots$ & 1 & 0.0100 & & $\ldots$ \\
\hline $24 / 27$ & 1 & 0.0100 & 2 & 0.0189 & 3 & 0.0300 & & $\ldots$ & & $\ldots$ & $28 / 30$ & 2 & 0.0200 & & & & $\ldots$ & 1 & 0.0100 & & $\ldots$ \\
\hline $24 / 28$ & 5 & 0.0500 & 8 & 0.0755 & 1 & 0.0100 & 5 & 0.0500 & & $\ldots$ & $28 / 31$ & 1 & 0.0100 & & & 1 & 0.0100 & & $\ldots$ & & $\ldots$ \\
\hline $24 / 29$ & 2 & 0.0200 & 1 & 0.0094 & 2 & 0.0200 & 3 & 0.0300 & & $\ldots$ & $28 / 32$ & & $\ldots$ & & & 1 & 0.0100 & & $\ldots$ & & $\ldots$ \\
\hline $24 / 31$ & 5 & 0.0500 & 1 & 0.0094 & 3 & 0.0300 & 2 & 0.0200 & & $\ldots$ & $28 / 34$ & & $\ldots$ & 2 & 0.018 & 3 & 0.0300 & & $\ldots$ & 1 & 0.0625 \\
\hline $24 / 32$ & & $\ldots$ & & $\ldots$ & & $\ldots$ & 1 & 0.0100 & & $\ldots$ & $28 / 40$ & & $\ldots$ & 1 & 0.009 & & $\ldots$ & & $\ldots$ & & $\ldots$ \\
\hline $24 / 33$ & & $\ldots$ & & $\ldots$ & & $\ldots$ & 1 & 0.0100 & & $\ldots$ & $29 / 34$ & 1 & 0.0100 & & & & $\cdots$ & & $\cdots$ & & $\ldots$ \\
\hline $24 / 34$ & 3 & 0.0300 & 4 & 0.0377 & 6 & 0.0600 & 2 & 0.0200 & 1 & 0.0625 & $31 / 31$ & 1 & 0.0100 & & & & $\ldots$ & & $\ldots$ & & $\ldots$ \\
\hline $24 / 36$ & & $\ldots$ & & $\ldots$ & & $\ldots$ & & $\ldots$ & 1 & 0.0625 & $31 / 32$ & & $\ldots$ & 2 & 0.018 & 1 & 0.0100 & & $\ldots$ & & $\ldots$ \\
\hline $24 / 40$ & & $\ldots$ & 2 & 0.0189 & 1 & 0.0100 & & $\ldots$ & & $\ldots$ & $31 / 34$ & 1 & 0.0100 & & & & $\ldots$ & 1 & 0.0100 & 1 & 0.0625 \\
\hline $25 / 25$ & & $\ldots$ & & $\ldots$ & 1 & 0.0100 & 1 & 0.0100 & & $\ldots$ & $31 / 40$ & & $\ldots$ & & & 1 & 0.0100 & & $\ldots$ & & $\ldots$ \\
\hline $25 / 26$ & & $\ldots$ & 1 & 0.0094 & & $\ldots$ & & $\ldots$ & & $\ldots$ & $32 / 34$ & & $\ldots$ & 2 & 0.018 & & $\ldots$ & & $\ldots$ & & $\ldots$ \\
\hline $25 / 28$ & 4 & 0.0400 & 1 & 0.0094 & 1 & 0.0100 & 1 & 0.0100 & & $\ldots$ & $34 / 34$ & 1 & 0.0100 & & & 1 & 0.0100 & & $\ldots$ & 1 & 0.0625 \\
\hline $25 / 31$ & 3 & 0.0300 & & $\ldots$ & & $\ldots$ & 1 & 0.0100 & & $\ldots$ & & & & & & & & & & & \\
\hline $25 / 34$ & & $\ldots$ & & $\ldots$ & & $\ldots$ & & $\ldots$ & 2 & 0.1250 & & & & & & & & & & & \\
\hline $26 / 26$ & & $\ldots$ & & $\ldots$ & & $\ldots$ & 1 & 0.0100 & & $\ldots$ & & & & & & & & & & & \\
\hline $26 / 29$ & & $\ldots$ & & $\ldots$ & & $\ldots$ & 1 & 0.0100 & & $\ldots$ & & & & & & & & & & & \\
\hline
\end{tabular}

$\ldots=$ allele not detected; $\mathrm{N}=$ Number of genotypes 
each population is calculated to be 136 for Benin, 153 for the Kenyan and Rwandan populations, 171 for Tanta and 66 for Cameroon.

The observed homozygosity for Benin, Cameroon, Kenya, Rwanda and Tanta were $0.1500,0.1880,0.0660,0.1500$ and 0.2800 , respectively. The corresponding heterozygosity values were $0.8500,0.8130,0.9340,0.8500$ and 0.7200 (Table 4 ). These values did not vary greatly from the expected ones. These high levels of heterozygosity are consistent with genetic diversity within African populations at other loci [46].

Figure 1 shows an $\mathrm{NJ}$ tree using Nei distances [47]. Four main clusters of populations can be discerned. Native Americans are seen in a cluster at one end of the tree. Caucasians are grouped in the adjacent clade. The Mapuche Argentin population segregated with the Caucasians. Hutz and collaborators also placed this population with Caucasians [33]. The following clade exhibits all the African groups as well as the Saudi Arabia and Moslem Arab populations. The last clade clusters the Oriental populations.

In the UPGMA depiction (Figure 2), there are two main branches after the bifurcation between the Andalusian population sample and all the other groups. These two branches have Denmark and Australia versus the rest of the populations in our study. Five clusters can be found here. Dubai Arabs head off the first cluster that incorporates other southwest Asian populations along with two southeast European populations, Tanta and Mapuche Argentina. Once again, Tanta appears to be most closely related to those countries across the adjoining Mediterranean Sea. This would suggest that southern European and southwest Asian countries neighboring the Mediterranean Sea contributed greatly to the genetic constitution of this group. The next cluster holds 
Figure 1. Neighbor Joining Tree

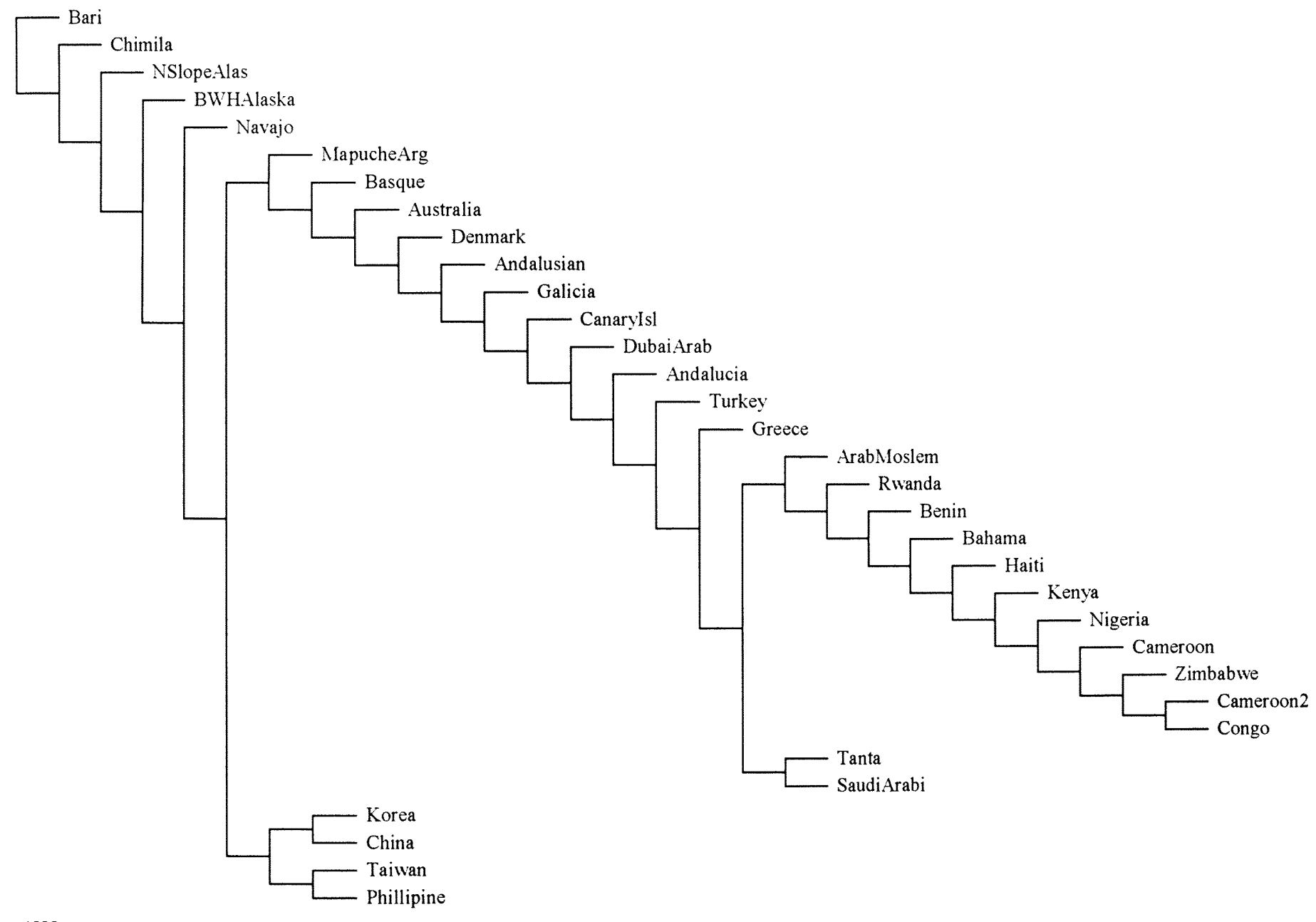


Figure 2. UPGMA Tree

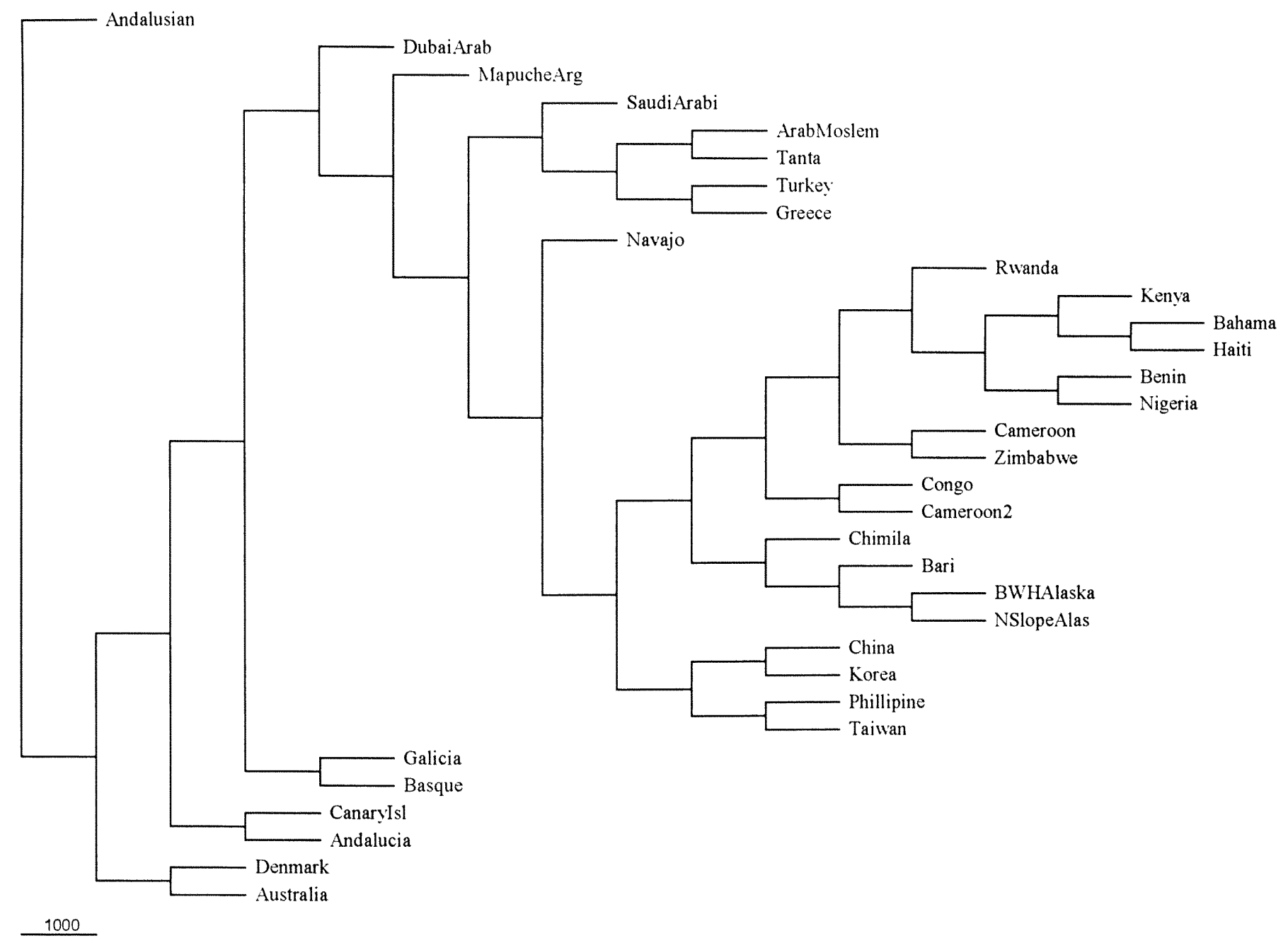


the Navajo population branched with the Africans and other Mongoloids. Lastly, the rest of the Caucasians are depicted.

Figure 3 shows a radial representation of the Maximum Likelihood Tree. This tree's primary bifurcation is between the southwest Asian populations and all other populations. All of the African groups are clustered together along with the Greek population. The Caucasians also segregate together. The Mongoloids make up the next assemblage. The bootstrap values in this figure indicate the likelihood that the depicted relationships are statistically significant. Bootstrap values greater than 50 percent (indicated as 500 or greater in the figure) are considered credible indicators of phylogenetic relationships.

Figure 4 displays the variations in all 27 alleles amongst the 33 populations included in the study. Each allele frequency is standardized upon input into the NTSys program. Two by two contingency tables were generated and eigenvalues and eigenvectors were calculated and then plotted. PC 1 indicated $21 \%$ of the variability while 14\% was held in PC 2. PC 1 shows delineation between African populations and all other populations. PC 2 separates west African populations from the rest of the African groups and the southeast Asians from the American Indian groups. There is also a separation between the southwest Asians and European groups.

The data represented in Table 5 indicates the results of tests for adherence to Hardy-Weinberg equilibrium expectations. A Fisher exact test, along with a Chi-Square test was performed. The Benin population did not conform to Hardy-Weinberg equilibrium expectations according to the Fisher exact test. Its probability (p) value was 
0.019. The Rwanda population was also shown to be marginally out of Hardy-Weinberg equilibrium by the Chi-Square test, with a p-value of 0.036 .

The G-test analysis of the 33 different populations (Table 6) provided data consistent with the other statistical tests previously mentioned. In the $2 \times 2$ comparisons, statistically insignificant values and low G-scores indicate homogeneity among populations, while statistically significant values with high G-scores are indicative of distinctiveness. G-score p-values are significant below 0.05 and indicate nonhomogeneity among populations. Generally, groups within the major races were not found to be significantly different. Notable exceptions were the Tanta population when compared to the sub-Saharan African groups and the Mapuche and Navajo populations when compared to other Native Americans. No significant differences were seen between the Tanta population and the Caucasoid groups. Similarly, the Mapuche and Navajo populations were not significantly different when compared to the Caucasian groups. 
Figure 3. Maximum Likelihood Tree with Bootstrap Values

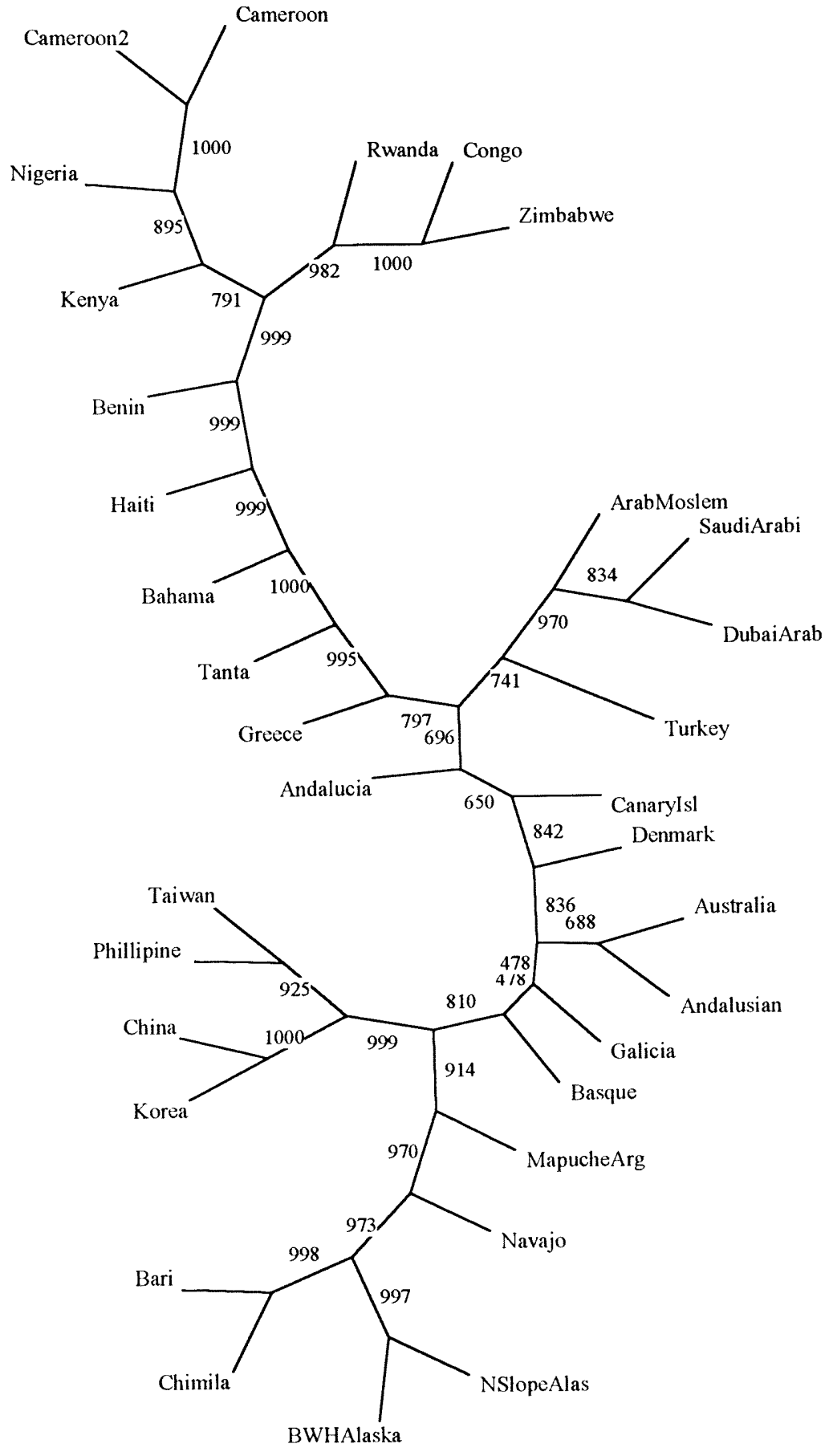


Figure 4. Principal Component Plot

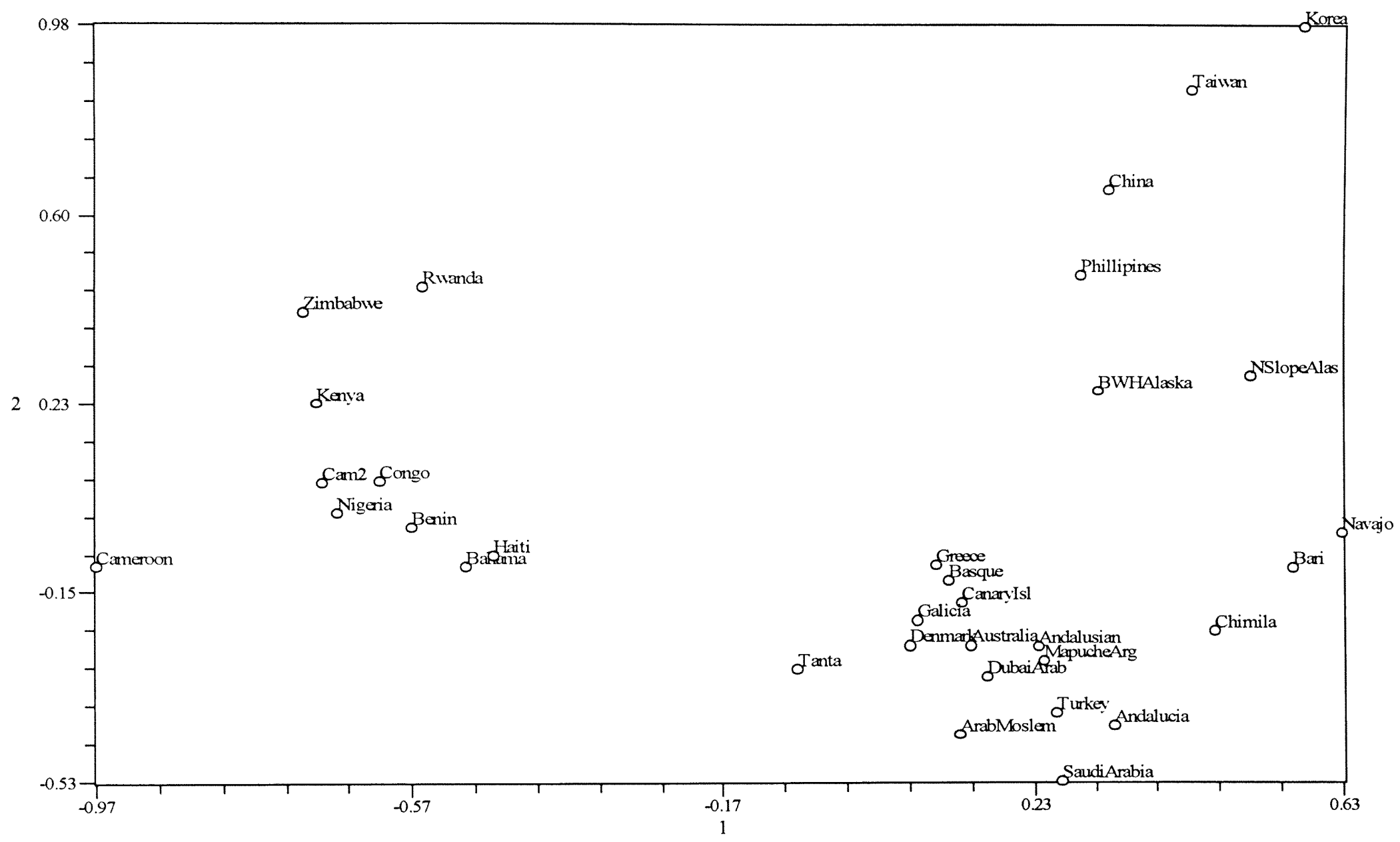


Table 4. Observed/Expected homozygosity and heterozygosity values

\begin{tabular}{lccccc}
\hline Population & N & $\begin{array}{c}\text { Observed } \\
\text { Homozygosity } \\
\text { \% }\end{array}$ & $\begin{array}{c}\text { Expected } \\
\text { Homozygosity } \\
\text { Unbiased (\%) }\end{array}$ & $\begin{array}{c}\text { Observed } \\
\text { Heterozygosity } \\
\%\end{array}$ & $\begin{array}{c}\text { Expected } \\
\text { Heterozygosity } \\
\text { Unbiased (\%) }\end{array}$ \\
\hline \hline Benin & 100 & 15 & 10.28 & 85 & 89.72 \\
Kenya & 106 & 6.6 & 11.18 & 93.4 & 88.82 \\
Rwanda & 100 & 15 & 10.87 & 85 & 89.13 \\
Tanta & 100 & 28 & 20.62 & 72 & 79.38 \\
Cameroon & 16 & 18.8 & 15.73 & 81.3 & 84.27
\end{tabular}

Table 5. Test for Hardy-Weinberg Equilibrium and Power of Discrimination \begin{tabular}{llll}
\hline Population & F & Fisher Exact & Chi Square Power of Discrimination
\end{tabular} (3200 shufflings) (3200 shufflings)

\begin{tabular}{lcccc} 
& & $(\mathrm{p})$ & $(\mathrm{p})$ & $\%$ \\
\hline \hline Benin & 100 & 0.019 & 0.109 & 96.98 \\
Kenya & 106 & 0.704 & 0.212 & 97.03 \\
Rwanda & 100 & 0.247 & 0.036 & 97.06 \\
Tanta & 100 & 0.049 & 0.377 & 93.48 \\
Caineroon & 16 & 0.879 & 0.492 & 92.19
\end{tabular}


Table 6. G Scores with probability values between all 33 populations

\begin{tabular}{|c|c|c|c|c|c|c|c|c|c|c|c|c|c|c|}
\hline & Anc & Ans & Ara & Aus & Bah & Bar & Bas & Ben & $\mathrm{BWH}$ & Cam & $\mathrm{Ca} 2$ & Can & $\mathrm{Chm}$ & Chn \\
\hline Andalucia & & 39.607 & 37.366 & 38.200 & 113.841 & 110.568 & 44.118 & 143.180 & 152.635 & 130.007 & 100.841 & 26.710 & 242.916 & 123.671 \\
\hline Andalusian & 0.904 & & 47.932 & 25.949 & 93.360 & 117.457 & 49.165 & 113.126 & 144.274 & 111.033 & 77.863 & 28.462 & 212.149 & 131.290 \\
\hline ArabMoslem & 0.895 & 0.732 & & 58.012 & 75.265 & 129.990 & 78.713 & 102.207 & 179.643 & 100.351 & 74.700 & 33.556 & 230.491 & 146.251 \\
\hline Australia & 0.937 & 1.000 & 0.322 & & 130.877 & 118.601 & 38.410 & 172.272 & 162.712 & 150.267 & 108.843 & 30.595 & 275.305 & 156.095 \\
\hline Bahama & 0.000 & 0.000 & 0.008 & 0.000 & & 151.487 & 140.302 & 17.390 & 162.303 & 31.186 & 43.138 & 98.566 & 231.274 & 163.705 \\
\hline Bari & 0.000 & 0.000 & 0.000 & 0.000 & 0.000 & & 103.104 & 167.631 & 65.014 & 141.848 & 106.582 & 113.677 & 65.327 & 117.950 \\
\hline Basque & 0.803 & 0.744 & 0.012 & 0.971 & 0.000 & 0.001 & & 178.190 & 141.540 & 152.011 & 110.707 & 42.610 & 274.587 & 152.081 \\
\hline Benin & 0.000 & 0.000 & 0.000 & 0.000 & 1.000 & 0.000 & 0.000 & & 176.910 & 31.453 & 37,415 & 121.300 & 251.940 & 218.647 \\
\hline Bethel-Wade & 0.000 & 0.000 & 0.000 & 0.000 & 0.000 & 0.009 & 0.001 & 0.000 & & 162.327 & 111.761 & 160.778 & 171.059 & 144.319 \\
\hline Cameroon & 0.000 & 0.000 & 0.000 & 0.000 & 0.967 & 0.000 & 0.000 & 0.958 & 0.000 & & 21.447 & 119.943 & 180.598 & 163.019 \\
\hline Cameroon2 & 0.000 & 0.000 & 0.000 & 0.000 & 0.677 & 0.000 & 0.000 & 0.812 & 0.000 & 1.000 & & 94.847 & 121.522 & 120.043 \\
\hline Canarylsland & 0.996 & 0.998 & 0.963 & 0.995 & 0.000 & 0.000 & 0.888 & 0.000 & 0.000 & 0.000 & 0.000 & & 233.634 & 109.141 \\
\hline Chimila & 0.000 & 0.000 & 0.000 & 0.000 & 0.000 & 0.004 & 0.000 & 0.000 & 0.000 & 0.000 & 0.000 & 0.000 & & 279.437 \\
\hline China & 0.000 & 0.000 & 0.000 & 0.001 & 0.000 & 0.000 & 0.001 & 0.000 & 0.000 & 0.000 & 0.000 & 0.000 & 0.000 & \\
\hline Congo & 0.000 & 0.000 & 0.000 & 0.000 & 0.176 & 0.000 & 0.000 & 0.425 & 0.000 & 0.934 & 0.993 & 0.000 & 0.000 & 0.000 \\
\hline Denmark & 0.994 & 0.995 & 0.294 & 1.000 & 0.000 & 0.000 & 0.993 & 0.000 & 0.000 & 0.000 & 0.000 & 0.997 & 0.000 & 0.001 \\
\hline DubaiArab & 0.674 & 0.723 & 0.975 & 0.575 & 0.000 & 0.000 & 0.157 & 0.000 & 0.000 & 0.000 & 0.000 & 0.935 & 0.000 & 0.000 \\
\hline Galicia & 0.902 & 0.990 & 0.146 & 0.976 & 0.000 & 0.000 & 1.000 & 0.000 & 0.000 & 0.000 & 0.000 & 0.998 & 0.000 & 0.001 \\
\hline Greece & 0.814 & 0.629 & 0.830 & 0.112 & 0.000 & 0.000 & 0.424 & 0.000 & 0.000 & 0.000 & 0.000 & 0.999 & 0.000 & 0.000 \\
\hline Haiti & 0.000 & 0.000 & 0.000 & 0.000 & 1.000 & 0.000 & 0.000 & 1.000 & 0.000 & 0.992 & 0.773 & 0.000 & 0.000 & 0.000 \\
\hline Kenya & 0.000 & 0.000 & 0.000 & 0.000 & 0.995 & 0.000 & 0.000 & 0.957 & 0.000 & 0.975 & 0.645 & 0.000 & 0.000 & 0.000 \\
\hline Korea & 0.000 & 0.000 & 0.000 & 0.001 & 0.000 & 0.000 & 0.001 & 0.000 & 0.000 & 0.000 & 0.000 & 0.000 & 0.000 & 0.998 \\
\hline Mapuche & 0.714 & 0.773 & 0.661 & 0.827 & 0.000 & 0.000 & 0.689 & 0.000 & 0.001 & 0.000 & 0.000 & 0.565 & 0.000 & 0.034 \\
\hline Navajo & 0.366 & 0.697 & 0.112 & 0.753 & 0.001 & 0.004 & 0.588 & 0.000 & 0.160 & 0.000 & 0.004 & 0.289 & 0.000 & 0.079 \\
\hline Nigeria & 0.000 & 0.000 & 0.009 & 0.000 & 1.000 & 0.000 & 0.000 & 1.000 & 0.000 & 1.000 & 0.960 & 0.000 & 0.000 & 0.000 \\
\hline NorthSlope & 0.000 & 0.000 & 0.000 & 0.000 & 0.000 & 0.024 & 0.000 & 0.000 & 0.319 & 0.000 & 0.000 & 0.000 & 0.000 & 0.000 \\
\hline Philippines & 0.000 & 0.000 & 0.000 & 0.000 & 0.000 & 0.000 & 0.000 & 0.000 & 0.000 & 0.000 & 0.000 & 0.002 & 0.000 & 0.243 \\
\hline Rwanda & 0.000 & 0.000 & 0.000 & 0.000 & 0.061 & 0.000 & 0.000 & 0.566 & 0.000 & 0.349 & 0.678 & 0.000 & 0.000 & 0.000 \\
\hline SaudiArabia & 0.054 & 0.001 & 0.329 & 0.001 & 0.000 & 0.000 & 0.001 & 0.000 & 0.000 & 0.000 & 0.000 & 0.005 & 0.000 & 0.001 \\
\hline Taiwan & 0.000 & 0.000 & 0.000 & 0.001 & 0.000 & 0.000 & 0.001 & 0.000 & 0.000 & 0.000 & 0.000 & 0.002 & 0.000 & 1.000 \\
\hline Tanta & 0.944 & 0.978 & 0.995 & 0.843 & 0.134 & 0.000 & 0.799 & 0.005 & 0.000 & 0.002 & 0.032 & 0.960 & 0.000 & 0.000 \\
\hline Turkey & 0.999 & 0.694 & 0.999 & 0.751 & 0.000 & 0.000 & 0.247 & 0.000 & 0.000 & 0.000 & 0.000 & 0.983 & 0.000 & 0.000 \\
\hline Zimbabwe & 0.000 & 0.000 & 0.000 & 0.000 & 0.227 & 0.000 & 0.000 & 0.546 & 0.000 & 0.922 & 0.891 & 0.000 & 0.000 & 0.000 \\
\hline
\end{tabular}

* G Scores are read with the vertical population columns at the side named first then the horizontal population columns at the top.

Probability values are read with the horizontal population columns at the top first, followed by the vertical population columns at the side. 
Table 6. G Scores with probability values between all 33 populations (Con't)

\begin{tabular}{|c|c|c|c|c|c|c|c|c|c|c|c|c|c|c|}
\hline & Con & Den & Dub & Gal & Gre & Hai & Ken & Kor & Map & Nav & Nig & NSA & Phi & Rwa \\
\hline Andalucia & 146.468 & 30.276 & 45.073 & 40.870 & 40.924 & 125.557 & 153.487 & 126.254 & 41.104 & 49.716 & 131.656 & 169.899 & 108.519 & 168.469 \\
\hline Andalusian & 111.572 & 31.112 & 45.934 & 30.928 & 47.290 & 106.217 & 135.584 & 138.957 & 43.099 & 43.987 & 109.624 & 157.981 & 120.831 & 142.548 \\
\hline ArabMoslem & 97.702 & 56.502 & 31.172 & 62.444 & 39.725 & 89.025 & 99.273 & 139.397 & 41.504 & 56.448 & 78.158 & 192.991 & 120.772 & 117.153 \\
\hline Australia & 171.063 & 20.131 & 50.561 & 34.893 & 65.816 & 155.570 & 191.049 & 168.103 & 41.717 & 42.383 & 156.745 & 172.030 & 147.608 & 223.569 \\
\hline Bahama & 56.588 & 121.455 & 97.947 & 115.624 & 91.023 & 9.918 & 26.481 & 130.172 & 84.808 & 80.533 & 20.551 & 227.178 & 159.925 & 65.632 \\
\hline Bari & 142.303 & 123.785 & 112.306 & 109.924 & 120.636 & 170.148 & 181.735 & 120.155 & 76.470 & 61.784 & 175.901 & 59.775 & 115.429 & 166.623 \\
\hline Basque & 179.871 & 32.600 & 62.875 & 25.339 & 53.222 & 167.673 & 209.024 & 158.468 & 45.585 & 46.337 & 168.817 & 159.639 & 128.989 & 223.925 \\
\hline Benin & 46.469 & 148.110 & 128.501 & 140.188 & 110.725 & 18.432 & 32.126 & 183.707 & 107.236 & 92.984 & 18.197 & 229.203 & 174.025 & 46.187 \\
\hline Bethel-Wade & 187.132 & 156.341 & 150.532 & 165.211 & 151.856 & 173.671 & 221.464 & 147.171 & 75,158 & 51.168 & 193.508 & 46.936 & 160.884 & 207.418 \\
\hline Cameroon & 33.435 & 136.752 & 113.046 & 128.385 & 105.311 & 27.216 & 30.811 & 137.238 & 90.682 & 112.572 & 22.213 & 206.225 & 157.942 & 50.547 \\
\hline Cameroon 2 & 29.011 & 101.393 & 81.840 & 84.684 & 93.787 & 37.972 & 42.955 & 100.573 & 77.951 & 71.980 & 32.567 & 118.487 & 106.827 & 41.962 \\
\hline CanaryIsland & 119.099 & 26.296 & 35.715 & 29.656 & 26.248 & 112.040 & 126.744 & 119.247 & 44.023 & 51.156 & 107.602 & 160.159 & 81.753 & 132.269 \\
\hline Chimila & 179.004 & 280.336 & 228.222 & 256.023 & 232.589 & 249.148 & 276.121 & 254.587 & 147.515 & 93.234 & 239.257 & 186.523 & 223.738 & 264.791 \\
\hline China & 186.581 & 152.396 & 131.487 & 148.493 & 117.489 & 180.447 & 221.648 & 32.800 & 72.374 & 63.737 & 194.085 & 159.601 & 60.732 & 230.538 \\
\hline Congo & & 161.252 & 117.265 & 130.518 & 108.194 & 55.783 & 52.436 & 152.371 & 125.675 & 102.051 & 35.139 & 191.858 & 142.699 & 38.328 \\
\hline Denmark & 0.000 & & 48.415 & 29.092 & 57.369 & 141.032 & 179.522 & 166.526 & 50.298 & 47.231 & 140.014 & 157.979 & 130.925 & 196.173 \\
\hline Dubai.Arab & 0.000 & 0.604 & & 40.832 & 45.101 & 110.938 & 126.084 & 130.129 & 45.437 & 55.877 & 102.245 & 145.521 & 128.256 & 122.977 \\
\hline Galicia & 0.000 & 1.000 & 0.851 & & 52.790 & 135.106 & 161.797 & 137.335 & 62.296 & 47.986 & 134.122 & 156.447 & 121.664 & 157.906 \\
\hline Greece & 0.000 & 0.260 & 0.595 & 0.384 & & 101.656 & 113.479 & 123.019 & 48.039 & 64.533 & 95.708 & 177.959 & 96.639 & 107.389 \\
\hline Haiti & 0.127 & 0.000 & 0.000 & 0.000 & 0.000 & & 21.703 & 142.693 & 95.959 & 93.759 & 20.347 & 238.417 & 171.147 & 67.002 \\
\hline Kenya & 0.294 & 0.000 & 0.000 & 0.000 & 0.000 & 1.000 & & 177.234 & 119.720 & 113.156 & 13.760 & 282.176 & 202.894 & 49.228 \\
\hline Korea & 0.000 & 0.000 & 0.000 & 0.000 & 0.000 & 0.000 & 0.000 & & 96.236 & 71.044 & 162.908 & 171.779 & 98.165 & 185.059 \\
\hline Mapuche & 0.000 & 0.468 & 0.611 & 0.121 & 0.365 & 0.000 & 0.000 & 0.000 & & 33.734 & 109.913 & 103.612 & 81.819 & 136.517 \\
\hline Navajo & 0.000 & 0.547 & 0.132 & 0.544 & 0.020 & 0.000 & 0.000 & 0.040 & 0.770 & & 101.281 & 53.518 & 56.840 & 105.015 \\
\hline Nigeria & 0.926 & 0.000 & 0.000 & 0.000 & 0.000 & 1.000 & 1.000 & 0.000 & 0.000 & 0.000 & & 234.626 & 166.791 & 30.837 \\
\hline NorthSlope & 0.000 & 0.000 & 0.000 & 0.000 & 0.000 & 0.000 & 0.000 & 0.000 & 0.000 & 0.135 & 0.000 & & 138.991 & 228.179 \\
\hline Philippines & 0.000 & 0.000 & 0.000 & 0.000 & 0.000 & 0.000 & 0.000 & 0.000 & 0.000 & 0.103 & 0.000 & 0.000 & & 173.564 \\
\hline Rwanda & 0.850 & 0.000 & 0.000 & 0.000 & 0.000 & 0.047 & 0.425 & 0.000 & 0.000 & 0.000 & 0.983 & 0.000 & 0.000 & \\
\hline Saudi.Arabia & 0.000 & 0.001 & 0.361 & 0.001 & 0.027 & 0.000 & 0.000 & 0.000 & 0.001 & 0.004 & 0.000 & 0.000 & 0.000 & 0.000 \\
\hline Taiwan & 0.000 & 0.000 & 0.000 & 0.000 & 0.000 & 0.000 & 0.000 & 0.693 & 0.072 & 0.304 & 0.000 & 0.000 & 0.995 & 0.000 \\
\hline Tanta & 0.000 & 0.971 & 0.894 & 0.918 & 0.989 & 0.010 & 0.001 & 0.000 & 0.617 & 0.211 & 0.017 & 0.000 & 0.000 & 0.000 \\
\hline Turkey & 0.000 & 0.687 & 0.766 & 0.433 & 0.973 & 0.000 & 0.000 & 0.000 & 0.788 & 0.145 & 0.000 & 0.000 & 0.000 & 0.000 \\
\hline Zimbabwe & 0.969 & 0.000 & 0.000 & 0.000 & 0.000 & 0.415 & 0.646 & 0.000 & 0.000 & 0.000 & 0.942 & 0.000 & 0.000 & 0.183 \\
\hline
\end{tabular}

* G Scores are read with the vertical population columns at the side named first then the horizontal population columns at the top.

Probability values are read with the horizontal population columns at the top first, followed by the vertical population columns at the side. 
Table 6. G Scores with probability values between all 33 populations (Con't)

\begin{tabular}{|c|c|c|c|c|c|}
\hline & Sau & Tai & Tan & Tur & $\mathrm{Zim}$ \\
\hline Andalucia & 65.720 & 103.030 & 36.641 & 26.075 & 222.281 \\
\hline Andalusian & 104.940 & 103.976 & 33.689 & 48.917 & 193.150 \\
\hline ArabMloslem & 51.768 & 107.591 & 29.973 & 27.009 & 159.421 \\
\hline Australia & 128.394 & 120.909 & 42.397 & 46.275 & 285.404 \\
\hline Bahama & 175.476 & 121.110 & 59.671 & 147.989 & 54.610 \\
\hline Bari & 176.081 & 122.218 & 133.725 & 119.068 & 191.230 \\
\hline Basque & 137.352 & 114.901 & 44.065 & 59.029 & 298.696 \\
\hline Benin & 218.823 & 145.400 & 75.585 & 152.219 & 44.397 \\
\hline Bethel-Wade & 296.155 & 126.507 & 148.347 & 117.485 & 245.616 \\
\hline Cameroon & 159.837 & 133.847 & 80.703 & 139.143 & 35.580 \\
\hline Cameroon2 & 110.536 & 97.612 & 65.794 & 106.624 & 36.584 \\
\hline CanaryIsland & 76.757 & 82.041 & 33.659 & 32.125 & 189.462 \\
\hline Chimila & 344.003 & 230.828 & 232.619 & 241.360 & 273.669 \\
\hline China & 308.712 & 23.733 & 129.085 & 137.124 & 272.791 \\
\hline Congo & 173.634 & 136.409 & 98.742 & 137.854 & 31.104 \\
\hline Denmark & 99.333 & 119.295 & 33.970 & 46.820 & 265.064 \\
\hline Dubai Arab & 47.847 & 120.199 & 37.462 & 45.368 & 207.168 \\
\hline Galicia & 97.748 & 117.993 & 38.109 & 54.235 & 233.191 \\
\hline Greece & 67.310 & 86.571 & 27.537 & 32.431 & 175.021 \\
\hline Haiti & 183.290 & 133.163 & 71.018 & 144.069 & 48.656 \\
\hline Kenya & 228.369 & 166.715 & 81.392 & 156.318 & 43.631 \\
\hline Korea & 277.317 & 48.672 & 133.840 & 149.635 & 213.142 \\
\hline Mapuche & 81.088 & 61.267 & 45.510 & 39.556 & 162.977 \\
\hline Navajo & 74.869 & 50.194 & 56.984 & 56.483 & 119.820 \\
\hline Nigeria & 164.973 & 143.123 & 70.595 & 131.079 & 34.258 \\
\hline NorthSlope & 303.174 & 140.537 & 179.921 & 186.407 & 296.454 \\
\hline Philippines & 209.880 & 27.685 & 111.666 & 115.354 & 208.422 \\
\hline Rwanda & 236.932 & 159.017 & 100.531 & 163.188 & 55.842 \\
\hline Saudi Arabia & & 213.788 & 46.167 & 49.613 & 342.655 \\
\hline Taiwan & 0.000 & \} $&{96.118} &{107.290} &{179.009} \\
{\hline \text { Tanta }} &{0.568} &{0.000} &{ } &{36.962} &{147.280} \\
{\hline \text { Turkey }} &{0.497} &{0.000} &{0.937} &{ } &{225.073} \\
{\hline \text { Zimbabwe }} &{0.000} &{0.000} &{0.000} &{0.000} &{ } \\
$\hline
\end{tabular}

Probability values are read with the horizontal population columns at the top first, followed by the vertical population columns at the side. 


\section{DISCUSSION}

To aid in our comparison of allelic frequencies, we generated allelic frequency graphs from Table 2 for each of our five African populations (Figures 5-9). Graphs were also created for African American and Caucasian samples from the United States (Figures $14 \& 15$ ) as well as the four previously data-banked African populations (Figures 10-13).

The African populations examined, including the African Americans, exhibited alleles 18,24, 28, 31 and 34, indicating genetic similarity. Exceptions to this include both Cameroon samples (Cameroon at allele 31 and Cameroon2 at allele 18), which may be due to sampling error. The two alleles most prevalent in US Caucasians were the same two that were found most frequently in the Tanta population.

The observed heterozygosity for the five African populations in our study was greater than $70 \%$. These heterozygosity values are indicative of the genetic diversity of this locus. Of the five African populations, the Kenya population appears to be the most diverse with a heterozygosity value of $93.4 \%$, compared to reported heterozygosity of $87.6 \%$ in the African American population [48]. The discriminatory power of the D1S80 locus was evident, with values above $90 \%$ for all five populations. Previous reports have indicated that the discrimination power for this locus is between 94 and $98 \%$ [49].

Of the five populations in our study, only the Benin population was significantly out of conformance to Hardy-Weinberg equilibrium expectations, with a Fisher exact test p-value of 0.019. Preferential amplification of specific alleles does not seem to be an explanation since a low number of samples tested were homozygous (15 of the 100 
samples). Our Theta values ranged from 0.009 in the Tanta population sample to 0.032 in the Benin samples. High values of theta indicates greater genetic substructure within a population. The overall theta-P value, indicating the variance present in the combination of all the alleles detected in the 5 African populations, for the D1S80 locus was 0.023 . This was comparable to published values of 0.019 [50]. The National Research Council II recommends the use of such a factor in all population studies. Recommendation 4.1 of this council's 1996 report suggests an inbreeding coefficient of 0.01 be used for United States populations, while an increased value of 0.03 would better suit more isolated populations [51].

The topology of the $\mathrm{NJ}$ tree illustrates the similarities between different populations by clustering them into clades. The Oriental and American Indian groups were separated from Caucasians and sub-Saharan Africans. Caucasians and sub-Saharan Africans are also separated from each other. This tree further discriminated between east Asians, with the two northern samples (Korea and China) grouped independently of the two southern ones (Taiwan and Philippine), and American Indians. The American Indian groups segregate together even though they may not be geographically close to each other, their origins ranging from North America to South America. The sample from Mapuche Argentina is genetically grouped with the Caucasians. The Tanta and Saudi Arabia populations segregated together indicating the historic influence that Saudi Arabian populations had on Tanta.

The UPGMA tree also clustered the populations based on their genetic similarities. Within this tree, the southeastern Europeans (Turkey and Greece) and the southwestern Asians (Saudi Arabia, Dubai Arab and Arab Moslem) were split from the 
Figure 5. Allelic Distribution Graph of the Benin Population

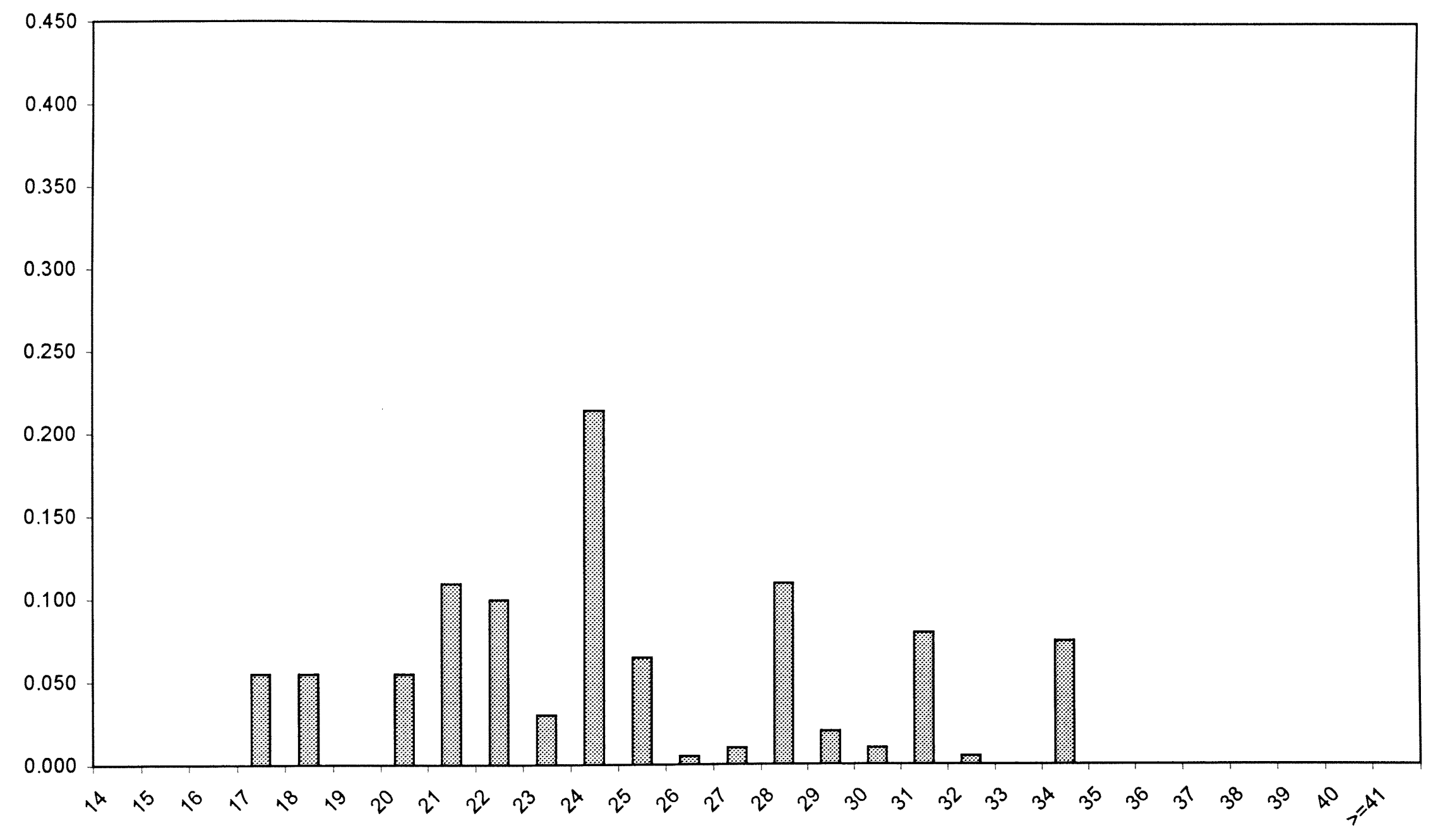


Figure 6. Allelic Distribution Graph of the Kenya Population

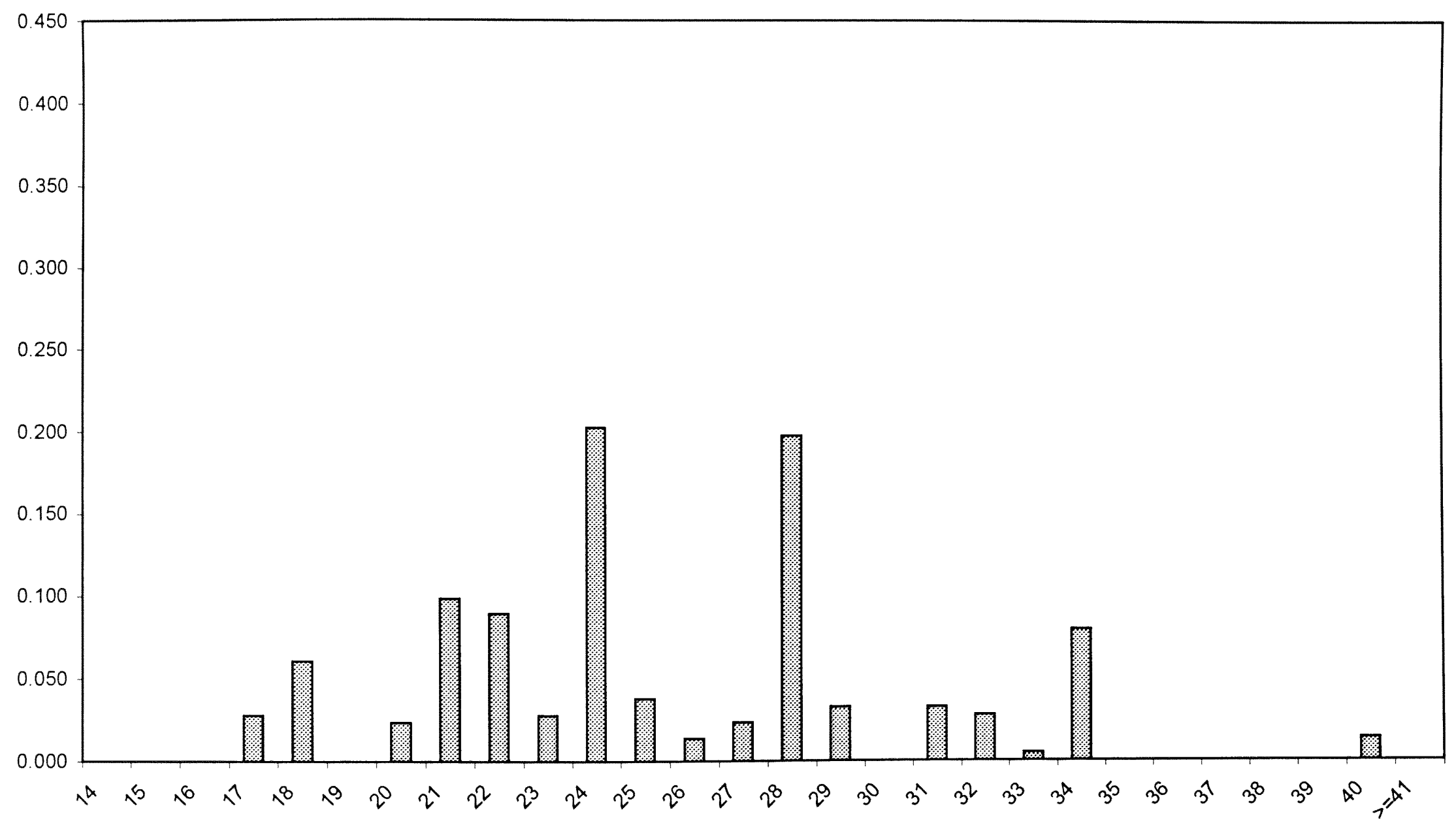


Figure 7. Allelic Distribution Graph of the Rwanda Population

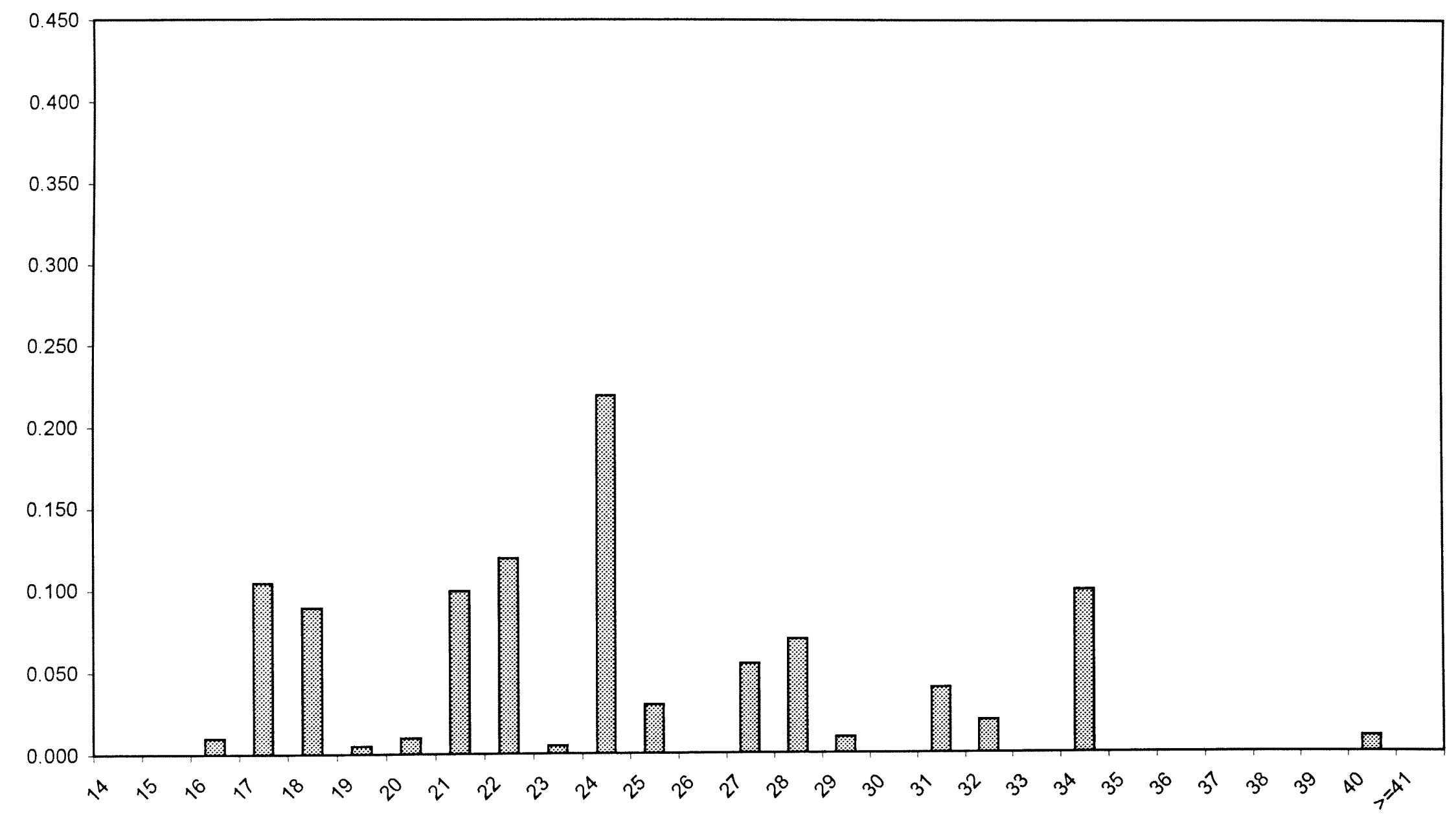




\section{Figure 8. Allelic Distribution Graph of the Tanta Population}

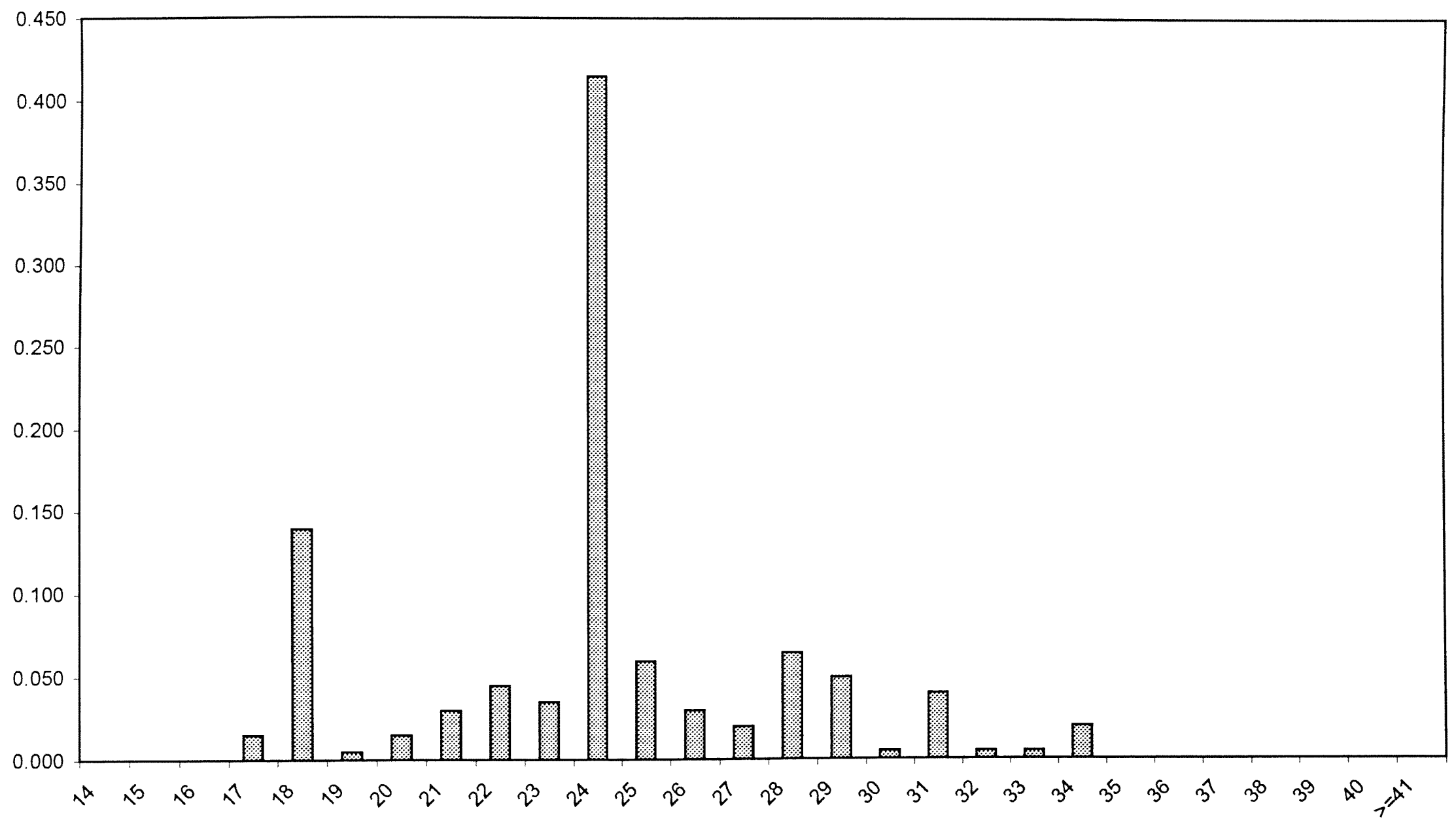


Figure 9. Allelic Distribution Graph of the Cameroon2 Population

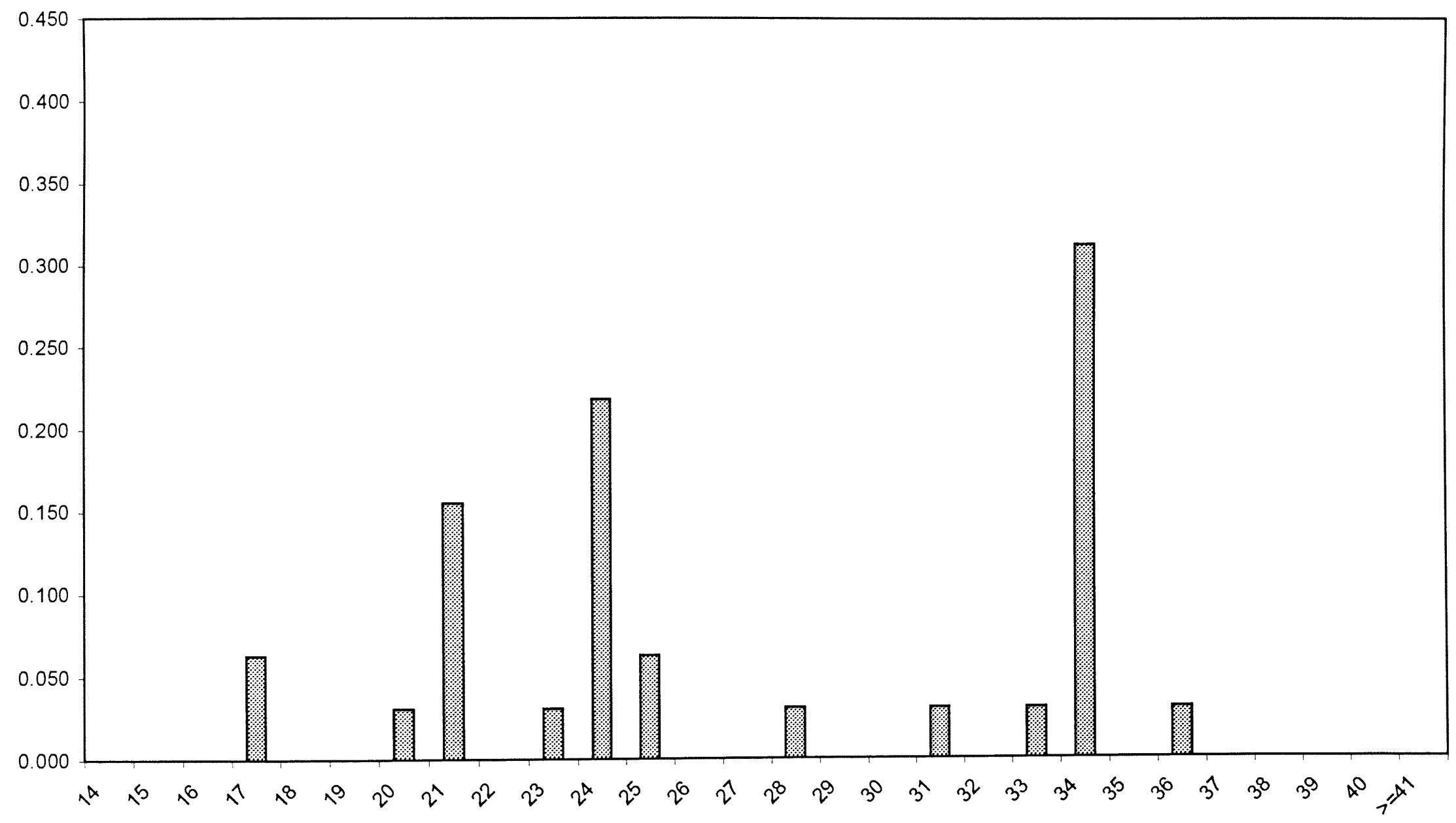


Figure 10. Allelic Distribution Graph of the Cameroon Population [26]

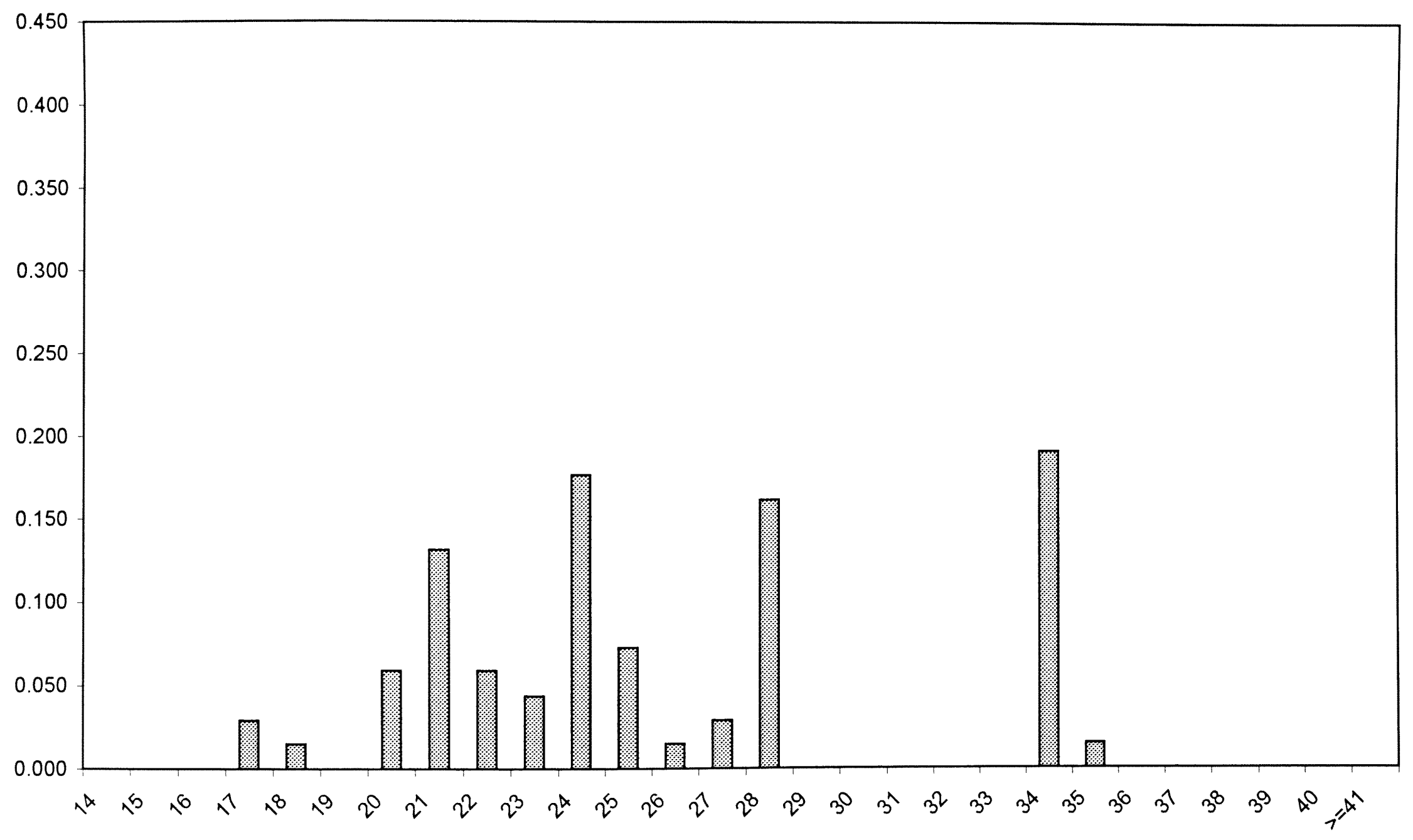


Figure 11. Allelic Distribution Graph of the Congo Population [26]

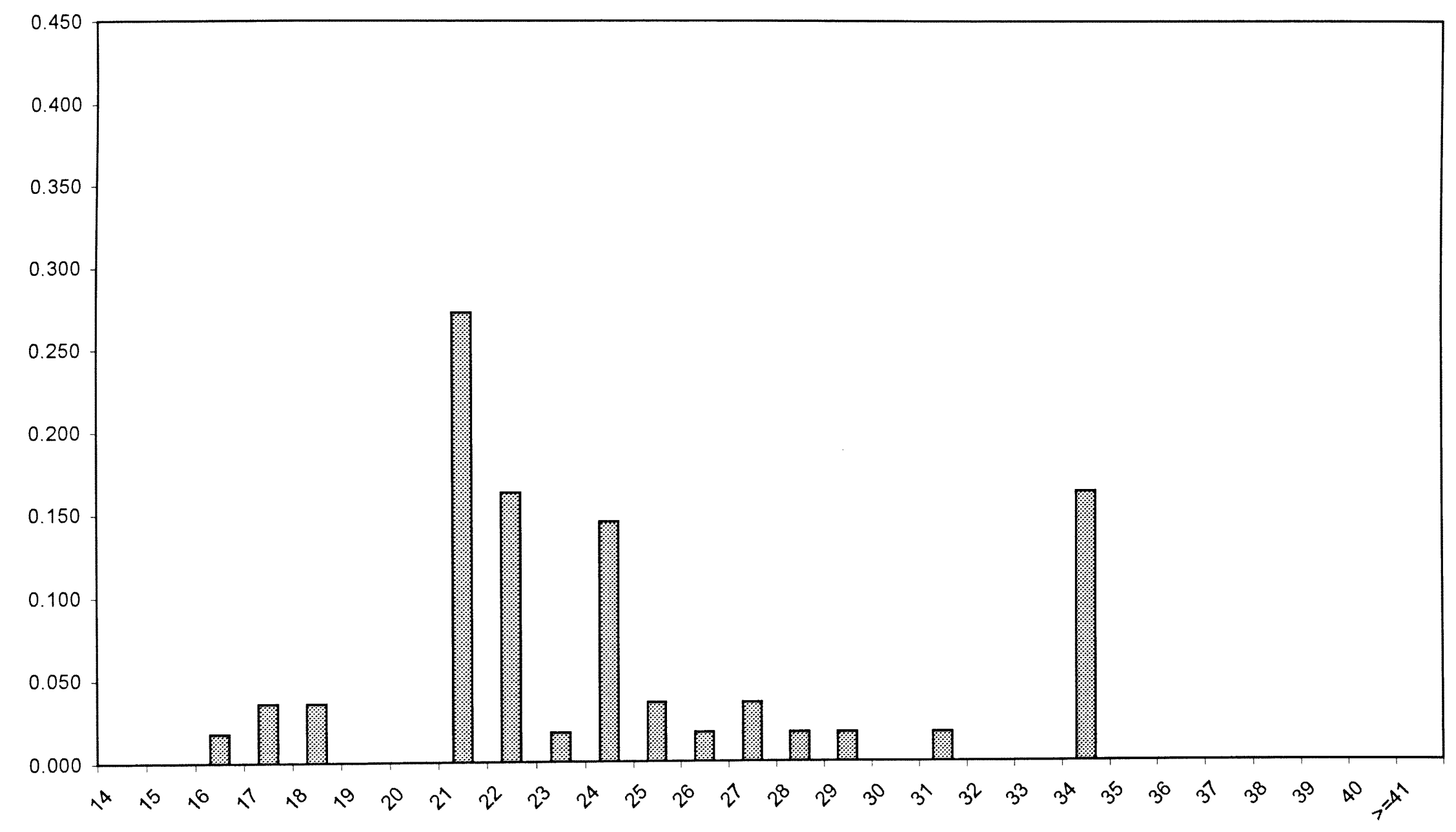


Figure 12. Allelic Distribution Graph of the Nigeria Population [28]

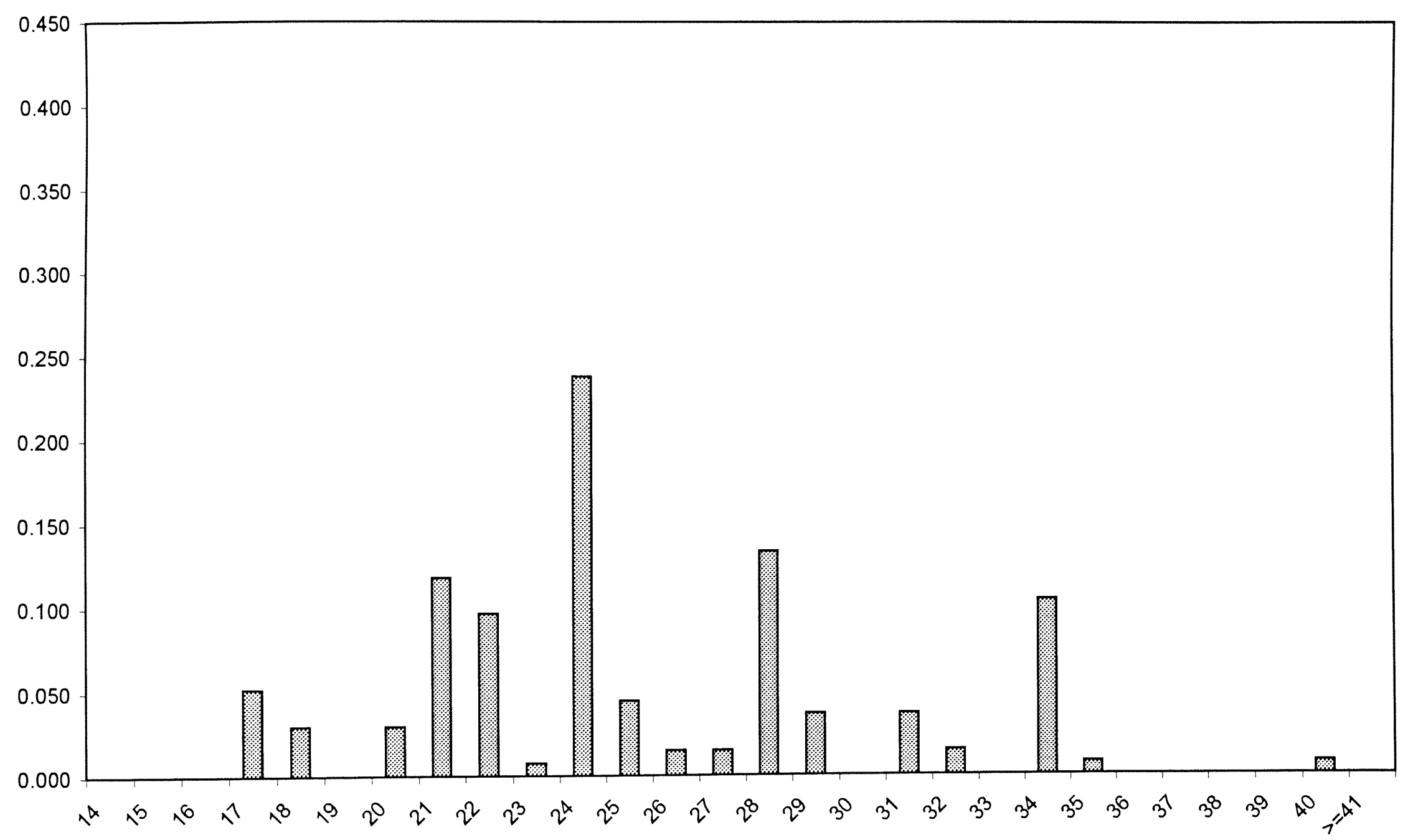


Figure 13. Allelic Distribution Graph of the Zimbabwe Population [28]

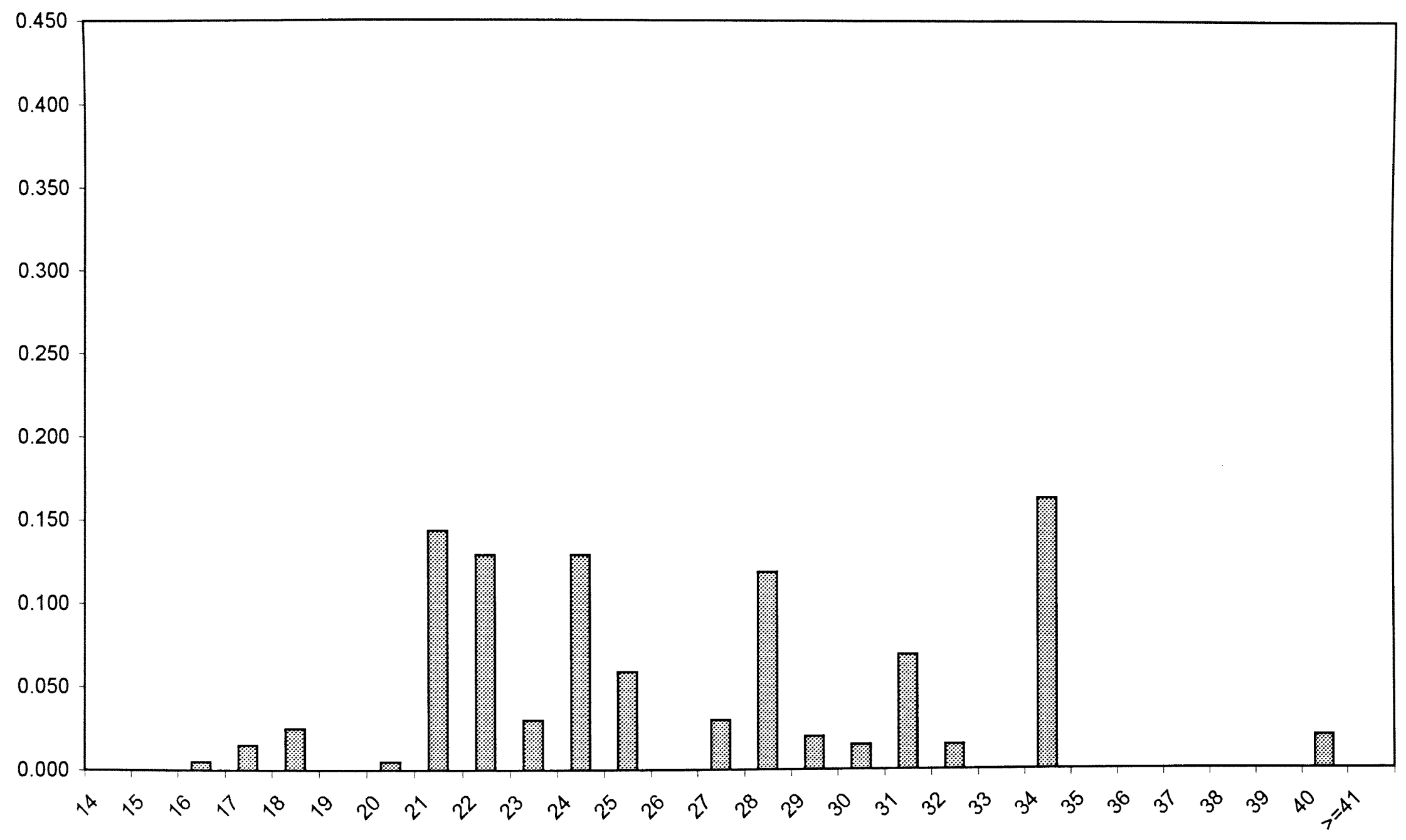


Figure 14. Allelic Distribution Graph of the African American Population [48]

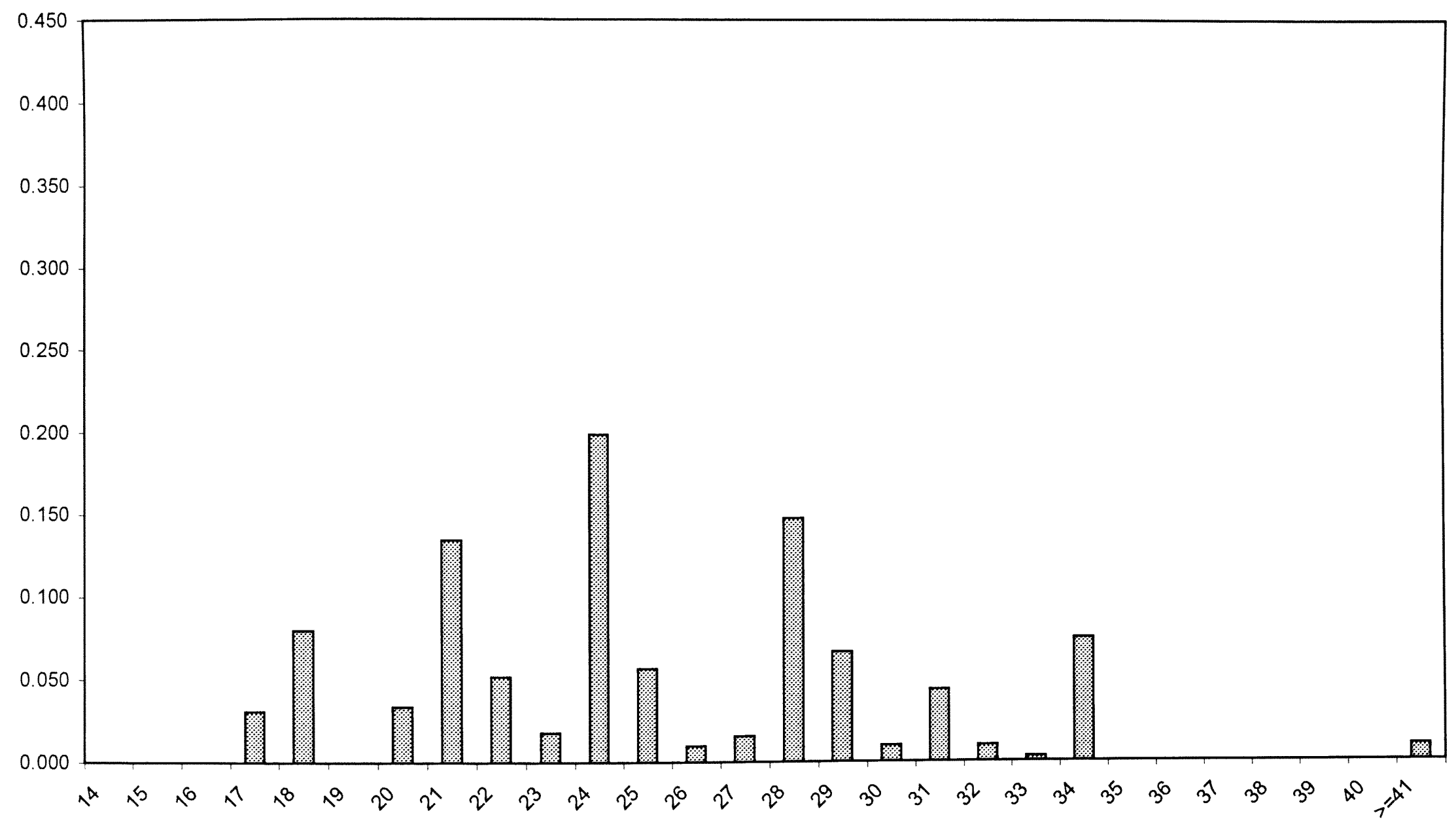


Figure 15. Allelic Distribution Graph of the Caucasian Population [48]

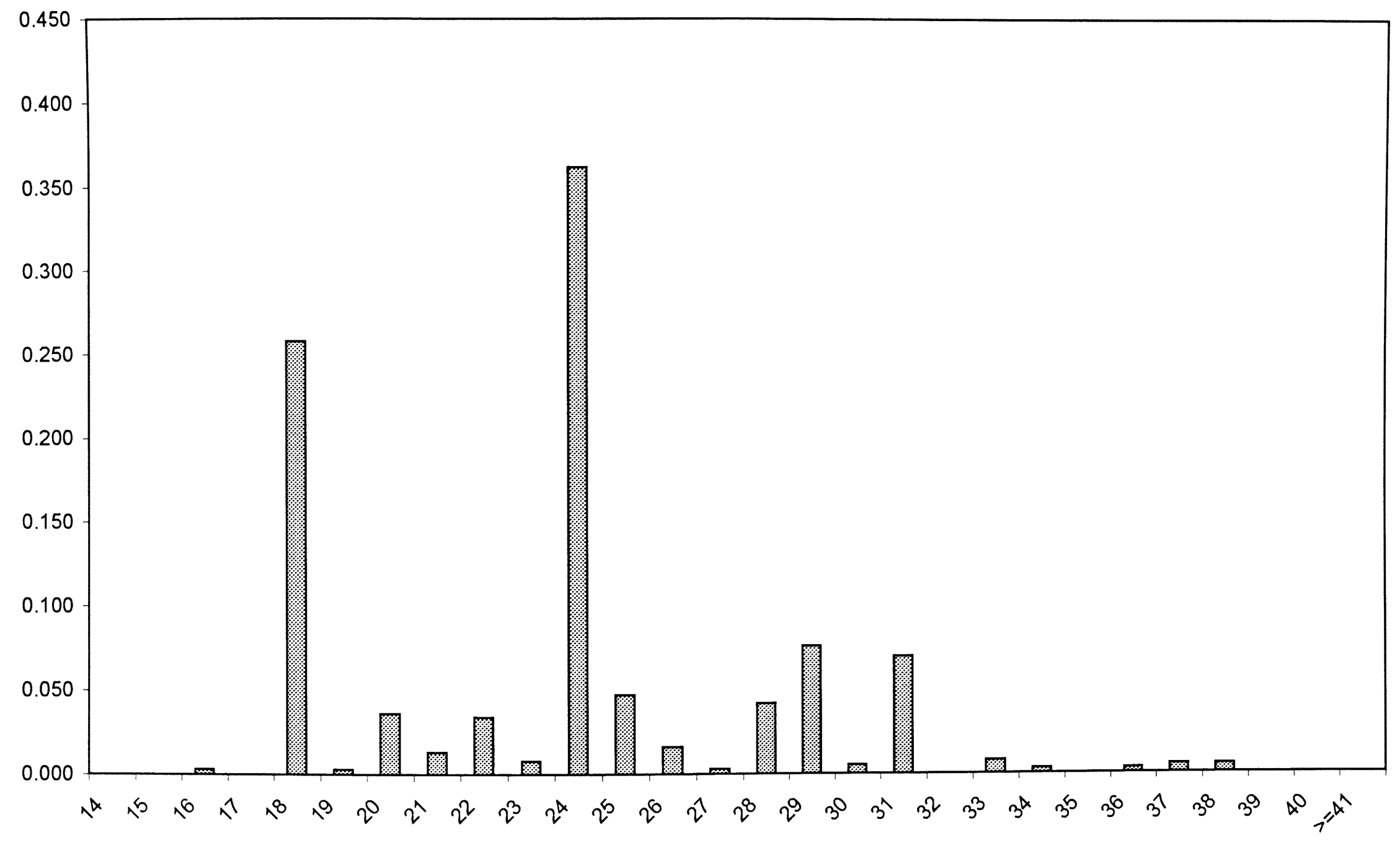


rest of the Caucasians. This is indicative of the variation detected between these groups. The Mapuche sample was more closely grouped to the southwest Asians than to the rest of the Caucasians. The two Alaskan groups show great homogeneity by segregating together. The east Asians are once again separately grouped into northern and southern regions.

Unlike the other two tree building methods, the ML dendrogram represented calculated distances based on the differences between the populations. Within this depiction, the southwest Asian groups (Saudi Arabia, Dubai Arab and Arab Moslem) are again separated from the other Caucasian populations. The east Asians are also shown to be distinct from the Native American groups even though they appear in the same cluster. The east Asians are segregated into northern and southern groups. The two Columbian Indian populations and the two Alaskan populations are shown to be genetically similar.

Throughout the previous analyses, consistent segregations were observed. The five African populations examined in our study were invariably grouped with the subSaharan African cluster, with the exception of the one Egyptian sample. The 16 Cameroon samples of our study segregated closely with the previously published Cameroon population [26].

The PC plot yielded a visual representation of allelic variability between the groups. This two-dimensional plot represented $35 \%$ of the total variation among the 27 alleles inputted. The x-axis of the PC clearly delineates the sub-Saharan African populations from the other populations. The north African population, Tanta, falls between this group and the Caucasians, nearer to the latter. Along the y-axis, the west Africans and west African derived populations are further segregated within the sub- 
Saharan cluster. The y-axis also separates east Asians from the American Indians and middle eastern populations from the rest of the Caucasians. Negroids showed the greatest variability between the alleles with a value of 0.5046 . This is indicated in the figure by the sparse arrangement of these populations. The Mongoloids represent the second most diverse group and are also sparsely gathered on the right side of the PC plot. The east Asians segregate together, as do the American Indians. Homogeneity is shown between the two Alaskan populations and the two Columbian populations that segregate near to each other. Similar to the NJ and UPGMA phylogenies, the Mapuche population segregates with the Caucasians, nearer to the southwest Asians. The Caucasian populations are clustered together closely denoting less diversity between the groups. However, segregation is still visible between the southwest Asians and the rest of the Caucasians. The Caucasians showed the least variability between their alleles with a value of 0.2662 .

The G-Test displayed homogeneity within the major races. It is in line with the other analyses that we performed in regard to the racial groupings of the populations. This analysis also grouped the Tanta and Mapuche populations with the Caucasians.

In this study we report on D1S80 databases from five African populations. The D1S80 locus provided us with a quick view of the phylogenetic relationships of the 33 populations examined. Although broad categorization of the populations was possible, clearly more polymorphic loci are required to provide for more significant comparisons. 


\section{CONCLUSION}

Our study has shown that this single locus is highly polymorphic. It was capable of separating 33 different populations into their constituent ethnic groups of Negroid, Mongoloid or Caucasoid. Each of the phylogenetic analyses performed indicated homogeneity between our African samples amongst themselves and in comparison to the few samples that previously existed from Africa.

The allelic distributions in our African populations were similar to that of other African and African derived populations in the literature. The Tanta population, which is believed to have been descendents of Caucasians, showed a similar distribution of alleles as the United States Caucasians [48].

Although D1S80 is an extensively studied locus, the majority of the data is focused on European populations (sparse database information currently exists for the following African nations: Cameroon, Congo, Nigeria and Zimbabwe). This study is the first to include greater than three African populations. A database useful for human differentiation has been created for four additional African populations. 


\section{REFERENCES}

[1.] Y. Nakamura, et al., Isolation and mapping of a polymorphic DNA sequence (pMCT118) on chromosome 1p(D1S80). Nucleic Acids Research. 16 (1988) 9364.

[2.] B. Budowle, et al., Multiplex Amplification and Typing Procedure for the Loci D1S80 and Amelogenin. J. Forensic Sci. 41 (1996) 660-663.

[3.] G. Duncan, Microvariation at the Human D1S80 locus. Doctoral Dissertation, FIU, 1996.

[4.] G. Duncan, et al., Human Phylogenetic Relationships According to the D1S80 locus. Genetica. 98 (1996) 277-287.

[5.] D. Betsch, J. Beard, D1S80 PCR with the $\$ 25$ Thermal Cycler. Biochemical Education. 27 (1999) 45-47.

[6.] L.L. Cavalli-Sforza, P. Menozzi, A. Piazza, The History and Geography of Human Genes, Princeton University Press, New Jersey, 1994. pp.73-83, 162-166.

[7.] G. P. Murdock, Africa: Its Peoples \& their culture history, McGraw-Hill Book Co, New York, 1959. pp. 111, 127, 392.

[8.] D. Levinson, Ethnic Groups Worldwide: A Ready Reference Handbook, Oryx Press, Phoenix, 1998. pp. 111, 115, 140, 152, 159-160.

[9.] C. Legum, ed., Africa Contemporary Record, Africana Publishing Co., London, 2000. pp. B377.

[10.] R. M. Maxon, Kenya. Microsoft Encarta Online. 2002.

[11.] C. C. Li, First Course in Population Genetics, Boxwood, Pacific Grove, 1976.

[12.] S. Cosso, R. Reynolds, Validation of the AmpliFLP D1S80 PCR Amplification Kit for Forensic Casework Analysis According to TWGDAM Guidelines. J. Forensic Sci. 40 (1995) 424-434.

[13.] Perkin-Elmer. AmplifLP D1S80 PCR Amplification Kit User Guide.

[14.] M. Tahir, et al., Distribution of D1S80 Alleles in the Bahrainian Population. J. Forensic Sci. 44 (1999) 1314-1315. 
[15.] H. Rangel-Villalobos, et al., Allele Frequency Distributions of Six Amp-FLPS (D1S80, APO-B, VWA, TH01, CSF1PO and HPRTB in a Mexican Population. Forensic Sci. Int. 105 (1999) 125-129.

[16.] I. Flores, et al., Population Data for Southern Spain and Canary Islands of HLADQA1, PM and D1S80 Loci. Forensic Sci. Int. 119 (2001) 116-118.

[17.] G. Duncan, et al., Microvariation at the Human D1S80 locus. Int. J. Legal Med. 110 (1996) 150-154.

[18.] I. Eisenbarth, et al., Description of Three New Alleles at the D1S80 (MCT118) Locus. J. Forensic Sci. 44 (1999) 841-842.

[19.] G. Watanabe, K. Shimizu,.DNA Sequence Analysis of Long PCR Amplified Products at the D1S80 Locus. Legal Medicine (In Press), (2001).

[20.] A. M. Gross, et al., Validation Studies for the Genetic Typing of the D1S80 Locus for Implementation into Forensic Casework. J. Forensic Sci. 42 (1997) 1140-1146.

[21.] I. Sanchez-Molina, R. Calvet, Allelic Frequencies for the HLA-DQA1, D1S80, HUMTH01, HUMTPOX, HUMCSF1PO and HUMVWA in Cantabria (Middle North Spain). J. Forensic Sci. 45 (2000) 167-169.

[22.] J. Soares-Vieira, et al., Allele and Genotype Frequencies for D1S80 Locus in a Brazilian Population Sample. J. Forensic Sci. 45 (2000) 696-697.

[23.] R. Deka, et al., Population genetic characteristics of the D1S80 locus in seven human populations. Human Genetics. 94 (1994) 252-258.

[24.] K. Kasai, et al., Amplification of a Variable Number of Tandem Repeats (VNTR) Locus (pMCT118) by the Polymerase Chain Reaction (PCR) and Its Application to Forensic Science. J. Forensic Sci. 35 (1990) 1196-2000.

[25.] A. Buchanan, et al., Long DOP-PCR of rare archival anthropological samples. Human Biology. 72 (2000) 911-925.

[26.] W. Araujo Da Silva Jr., et al., Genetic Diversity of Two African and Sixteen South American Populations Determined on the Basis of Six Hypervariable Loci. Am. J. Phys. Anthropol. 109 (1999) 425-437.

[27.] M. Lorente, et al., Spanish Population Data on Seven Loci:D1S80, D17S5, HUMTH01, HUMVWA, ACTBP2, D21S11 and HLA-DQA1. Forensic Sci. Int. 86 (1997) 163-171 
[28.] B. Peterson, et al., World Population Data for the HLA-DQA1, PM and D1S80 Loci with Least \& Most Common Profile Frequencies for Combinations of Loci Estimated Following NRC II Guidelines. J. Forensic Sci. 45 (2000) 118-146.

[29.] S. Gutowski, et al., Statistical Analysis of an Australian Population for the Loci Gc, HLA-DQA1, D1S80 and HUMTH01. Forensic Sci. Int. 76 (1995) 1-6.

[30.] M. Walkinshaw, et al., DNA Profiling in Two Alaskan Native Populations Using HLA-DQA1, PM, and D1S80 Loci. J. Forensic Sci. 41 (1996) 478-484.

[31.] A. Alkhayat, et al., Population Data on the PCR-Based Loci LDLR, GYPA, HBGG, D7S8, Gc, HLA-DQA1, and D1S80 from Arabs from Dubai. Forensic Sci. Int. 81 (1996) 29-34.

[32.] M. A. Cariolou, et al., Greek Cypriot Allele and Genotype Frequencies for Amplitype PM-DQA1 and D1S80 Loci. J. Forensic Sci. 43 (1998) 661-664.

[33.] M. Hutz, et al., D1S80 locus variability in South American Indians. Annals of Human Biology. 24 (1997) 249-255.

[34.] S. C. Halos, et al., Philippine Population Database at Nine Microsatellite Loci for Forensic and Paternity Applications. Forensic Sci. Int. 101 (1999) 27-32.

[35.] M. A. Tahir, et al., Distribution of Amplified Fragment Length Polymorphism D1S80 Alleles in a Saudi Arabian Population. J. Forensic Sci. 44 (1999) 1159.

[36.] A. H. Cakir, et al., Distribution of Amplified Fragment Length Polymorphism D1S80 Alleles in Turkish Population. J. Forensic Sci. 44 (1999) 1002.

[37.] J. Felsenstein, PHYLIP (Phylogeny Inference Package). Version 3.5c. Distributed by the author, Department of Genetics, University of Washington, Seattle.

[38.] F.J. Rohlf, Numerical Taxonomy System (NTSys pc). Version 2.1. Exeter Publishing Ltd., Setauket, NY.

[39.] G. Carmody, G-Test. Distributed by the author, Department of Biology, Carleton University, Ottawa.

[40.] S. Sherry, M. Batzer, Modeling Human Evolution - To Tree or Not to Tree? Genome Research. 7 (1997) 947-949.

[41.] P.O. Lewis, D. Zaykin, Genetic Data Analysis: Computer program for the analysis of allelic data. Version $1.0(\mathrm{~d} 16 \mathrm{c})$. Free program distributed by the authors over the internet from http://lewis eeb.uconn edu/lewishome/software.html. (2001) 
[42.] J.W. Lee, et al., Evaluation of DNA Match Probability in Criminal Case. Forensic Sci. Int. 116 (2001) 139-148.

[43.] K. Monson, B. Budowle, Effect of Reference Database on Frequency Estimates of Polymerase Chain Reaction (PCR)-Based DNA Profiles. J. Forensic Sci. 43 (1998) 483488.

[44.] Population Genetics of Gene Flow. 2002, Wake Forest University. Accessed through www.wfu.edu/users/sipet $00 \mathrm{~g} /$ populationgenetics. html.

[45.] R. Duran, M. Ruiz-Garcia, Genetic Population History Relationships of the Population of Bogota, Columbia, by Using the D1S80, VWA, and TH01 Molecular Markers. Am. J. Hum. Biol.. 13 (2001) 374-383.

[46.] W. S. Watkins, et al., Patterns of Ancestral Human Diversity: An Analysis of AluInsertion and Restriction-Site Polymorphisms. Am. J. Hum. Genet. 68 (2001) 738-752.

[47.] M. Nei, Genetic Distance Between Populations. The American Naturalist. 106 (1972) 283-292.

[48.] B. Budowle, et al., United States Population Data on the Multiplex Short Tandem Repeat Loci - HUMTH01, TPOX, and CSF1PO - and the Variable Number Tandem Repeat Locus D1S80. J. Forensic Sci. 42 (1997) 846-849.

[49.] AmpliFLP D1S80 PCR Amplification Kit Product Detail. 2001, Applied Biosystems.

[50.] W. Klitz, et al., Analysis of Genotype Frequencies and Interlocus Association for the PM, DQA1, and D1S80 Loci in Four Populations. J. Forensic Sci. 45 (2000) 10091015.

[51.] The Committee on DNA Forensic Science, The Evaluation of Forensic DNA Evidence, National Academy Press, Washington D.C., 1996. p.5. 